\title{
MicroRNA regulation and its effects on cellular transcriptome in Human Immunodeficiency Virus-1 (HIV-1) infected individuals with distinct viral load and CD4 cell counts
}

Karolina Duskova ${ }^{1}$, Pruthvi Nagilla ${ }^{1}$, Hai-Son Le ${ }^{2}$, Priyadarshini lyer ${ }^{1}$, Anbupalam Thalamuthu ${ }^{3}$, Jeremy Martinson ${ }^{1}$, Ziv Bar-Joseph², William Buchanan ${ }^{1}$, Charles Rinaldo ${ }^{1}$ and Velpandi Ayyavoo ${ }^{1^{*}}$

\begin{abstract}
Background: Disease progression in the absence of therapy varies significantly in HIV-1 infected individuals. Both viral and host cellular molecules are implicated; however, the exact role of these factors and/or the mechanism involved remains elusive. To understand how microRNAs (miRNAs), which are regulators of transcription and translation, influence host cellular gene expression (mRNA) during HIV-1 infection, we performed a comparative miRNA and mRNA microarray analysis using PBMCs obtained from infected individuals with distinct viral load and CD4 counts.

Methods: RNA isolated from PBMCs obtained from HIV-1 seronegative and HIV-1 positive individuals with distinct viral load and CD4 counts were assessed for miRNA and mRNA profile. Selected miRNA and mRNA transcripts were validated using in vivo and in vitro infection model.

Results: Our results indicate that HIV-1 positive individuals with high viral load (HVL) showed a dysregulation of 191 miRNAs and 309 mRNA transcripts compared to the uninfected age and sex matched controls. The miRNAs miR19b, 146a, 615-3p, 382, 34a, 144 and 155, that are known to target innate and inflammatory factors, were significantly upregulated in PBMCs with high viral load, as were the inflammatory molecules CXCL5, CCL2, IL6 and IL8, whereas defensin, CD4, ALDH1, and Neurogranin (NRGN) were significantly downregulated. Using the transcriptome profile and predicted target genes, we constructed the regulatory networks of miRNA-mRNA pairs that were differentially expressed between control, LVL and HVL subjects. The regulatory network revealed an inverse correlation of several miRNA-mRNA pair expression patterns, suggesting HIV-1 mediated transcriptional regulation is in part likely through miRNA regulation.
\end{abstract}

Conclusions: Results from our studies indicate that gene expression is significantly altered in PBMCs in response to virus replication. It is interesting to note that the infected individuals with low or undetectable viral load exhibit a gene expression profile very similar to control or uninfected subjects. Importantly, we identified several new mRNA targets (Defensin, Neurogranin, AIF) as well as the miRNAs that could be involved in regulating their expression through the miRNA-mRNA interaction.

\footnotetext{
* Correspondence: velpandi@pitt.edu

'Department of Infectious Diseases and Microbiology, Graduate School of Public Health, University of Pittsburgh, 425 Parran Hall, 130 Desoto Street, Pittsburgh, PA 15261, USA

Full list of author information is available at the end of the article
} 


\section{Background}

HIV-1 infected individuals show remarkable variation in virus replication and disease progression [1,2]. Recent studies support the notion that the host cellular gene expression profile (the transcriptome), in the context of virus infection, is directly correlated with disease patterns [3-5]. The gene expression pattern associated with HIV-1 infection in cells is likely controlled by host genetics and external factors, leading to dysregulated antiviral activity, inflammatory response and disease progression. The replication, spread, and immune evasion of the virus and the progression of disease depend on host cellular transcription and gene regulation in virus-specific target cells and bystander cells [6-8]. As virus replication is dependent on host cellular machinery, high viral load (HVL) augments cell destruction and gene regulation, whereas low viral load (LVL) or undetectable viral load promotes latency and possibly immune control. Further, increased virus production also results in release of free extracellular (cell- and virusfree), virion-associated, and cell-associated viral antigens, as well as infectious and noninfectious virus particles that could potentially alter bystander cell transcriptome and destruction. Consistent with this scenario, HIV-1 virus with defective expression of viral proteins such as Nef, Vpr, Gag, and Pol is shown to induce differential gene expression [9-11].

Host factors bearing on the outcome of HIV-1 infection include genetic elements such as HLA alleles, polymorphisms in HIV-1 receptors and coreceptors, and genes involved in innate and adaptive immune responses [12-15]. Following the landmark discovery of the CCR5$\triangle 32$ mutation that protects against HIV-1 infection [16-18], many other genetic variants have been shown to affect HIV-1 infection and AIDS pathogenesis [12,19,20]. It is likely that, in addition to incomplete immunological control, host genetic variation and differences in gene expression in the infected host cells may also contribute to the differential disease pattern [21-23]. Along these lines, attempts were made to identify host cellular proteins associated with HIV-1 infection [24-27]. There is limited information, however, regarding the regulation of host cellular genes at the transcriptional, posttranscriptional, and translational levels.

Previous studies have shown that HIV-1 infection differentially regulates host cellular factors responsible for inflammation, immune response, cell cycle/proliferation and apoptosis, suggesting that differential gene expression in infected individuals either accelerates disease progression or enhances resistance to the development of disease [28-30]. Recent discoveries have emphasized a central role for the new class of small non-coding RNA in gene expression controlling growth, development, and immune response in vivo [31-33]. Regulation of gene expression by miRNA occurs primarily at the posttranscriptional level [34,35]. Recent studies have shown that miRNA have a unique expression profile in cells of the innate and adaptive immune systems, CNS, and cancers [36-39]. Based on these observations, we hypothesize that pathogens including viruses could potentially modulate host cellular transcription at multiple levels by targeting various factors including miRNAs.

Studies previously have evaluated the expression of either miRNA or mRNA using PBMCs, CD4+ Tlymphocytes, CD8+ T-lymphocytes, monocytes and/or brain tissue from HIV-1 infected subjects [4,40-48]. Results from these studies have identified a number of cell specific miRNAs that are differentially regulated by HIV1 infection. Similarly regulated mRNA transcripts targeting IFN, cytokine-cytokine receptor signaling, apoptosis and MAPK signaling were also regulated by HIV-1 infection. However, in the case of HIV-1 infection, few combined miRNA and mRNA analyses using RNA isolated from the same sample or at the same time point have been conducted, except for the recent studies by Zhou et al [49] and Chang et al [50], using HIV-1 subjects brain tissue and infected CD4+ T cells, respectively. These studies provided the first line of evidence for a functional relationship between miRNA and mRNA in the context of HIV-1. In an effort to understand miRNA-mRNA interactions in the host during HIV-1 infection, we performed comparative global miRNA and mRNA microarray profiling in PBMCs derived from HIV-1 infected individuals with high and low or undetectable viral load and compared these with age and sex matched uninfected controls. Our results indicate that individuals with high viral load showed significant differences in both their miRNA and mRNA profiles compared to subjects with low viral load. In HIV-1 positive subjects with $>45,000$ copies $/ \mathrm{ml}$ of viral RNA, a high proportion of miRNAs (178/191) showed increased expression (with $>2$-fold change, $\mathrm{p}$ value of $<0.05$ ), whereas the mRNA profile showed opposing results with a higher number of genes (184/308) being downregulated. The regulatory network analysis revealed that several host cellular factors, implicated in HIV-1 disease progression, might be modulated through miRNA regulation of gene expression during disease development. Taken together, these results provide evidence that the miRNA profile could be an early indicator of HIV-1 induced host cellular dysfunction.

\section{Results}

miRNA profiling in control versus HIV-1 positive groups

RNA isolated from HIV-1 subjects with high and low viral loads, and from control subjects were subjected to miRNA profiling and data analyses to identify the differentially expressed miRNA within these groups. RNA 
isolated from PBMCs was tested for RNA quality and integrity by Bioanalyzer and by Taqman assays for endogenous control miRNAs and mRNA species. Specifically, the samples were tested for RNU48, miR-26b, or $\mathrm{U6}$, and assessed by the absolute $\mathrm{Ct}$ value of $<25$. The expression profile of 754 host cellular miRNAs (excluding endogenous controls) was assessed in PBMCs obtained from HIV-1 negative and HIV-1 positive subjects. Results from high and low viral load groups were compared with the uninfected control group as well as within the HIV-1 positive groups. Differentially regulated miRNAs between these groups were analyzed using two different software packages with the appropriate settings required for each software to maximize the confidence. The first program used was DataAssist with default settings (maximum allowable Ct value was 40 ), as suggested by the manufacturer. The Benjamini-Hochberg False Discovery Rate adjustment was applied for multiple comparison corrections. Our results indicate that 38, 244 and 183 miRNAs were differentially regulated in control versus LVL, control versus HVL, and LVL versus HVL respectively, with at least a 2 -fold change and $\mathrm{p}$ value $<0.05$ (Table 1 ).

For the StatMiner analysis, miRNAs that show a raw $\mathrm{Ct}$ value of $<36$, and miRNAs that are expressed in $>25 \%$ of the subjects in each group, were included as suggested [51]. This resulted in the elimination of 177, 173 and 160 miRNAs from each comparison (control vs LVL; Control vs HVL; LVL vs HVL) respectively. The remaining miRNA were assessed using a statistical package to identify the significantly regulated miRNA within and between these groups. Our results indicate that HIV-1 infection differentially regulated expression of several miRNAs $(+/-1.72$-fold with $\mathrm{p}<0.05$ using the Benjamini-Hochberg method within each group (Additional file 1: Table S1)). The level of expression in infected subjects was compared with that in uninfected controls, and fold differences were calculated based on normalization with endogenous control, U6 snRNA/ mammU6, as suggested by the manufacturer. Our results indicate that 41, 218 and 182 miRNAs were differentially regulated with $+/$ - 2 -fold change and $p<0.05$ in control versus LVL, control versus HVL and LVL versus HVL, respectively (Table 1). To further increase the confidence, miRNAs that were identified as differentially regulated by both programs were assessed, and 21, 191 and 158 of miRNAs were commonly found in both analyses $(\mathrm{p}<0.05)$ of control versus $\mathrm{LVL}$, control versus HVL and LVL versus HVL, respectively. However, as the significance increases to $\mathrm{p}<0.01$ both analyses identified more than $70 \%$ of miRNAs commonly.

\section{Differential regulation of miRNA in HIV-1 subjects with a broad range of viral load and CD4 counts}

Among the 754 miRNAs tested, 21 miRNAs were differentially regulated in low viral load individuals compared to uninfected controls using two different softwares (Table 1). Of these, 2 miRNAs were downregulated and 19 miRNAs were upregulated (fold change ranging from 3.09 to 20.48). However, in the HVL group, the number of differentially regulated miRNAs was significantly higher. A total of 191 miRNAs were differentially regulated in HVL subjects compared to uninfected controls. Of these, 13 were downregulated (fold change ranging from -2.05 to -89.82 ) and 178 were upregulated. Similarly, we identified 158 miRNAs that were differentially regulated when we compared the LVL and HVL groups. Of these, 27 miRNAs were downregulated and 131 miRNAs were upregulated, with the fold change ranging from 1.73 to 6460.41 . Together these results indicate that HIV-1 infection upregulated expression of several miRNAs and the effect was more significant in high viral load group compared to low viral load group.

Figure 1A shows the miRNAs that are specific to each group, as well as the overlap between the groups. When uninfected controls (CT) are compared with HIV-1 infected samples, 17 miRNAs are commonly dysregulated in the HIV-1 infected subjects (LVL and HVL combined). Among the 21 miRNAs that are differentially regulated between the CT vs. LVL groups, 3 were unique to the LVL group, whereas when the CT and HVL groups are compared, 62 of the 191 differentially regulated miRNAs were specific to the HVL group. Similarly, we also compared all three groups and found that 4 miRNAs are commonly dysregulated in all three groups. Further examination indicates that miR-1275, miR-483-5p, and miR-650 show similar upregulation in the HVL group compared to either the CT or LVL group, whereas

Table 1 Differentially regulated miRNA in low and high viral load groups compared to uninfected control group using two different software with statistical significance

\begin{tabular}{|c|c|c|c|c|c|c|}
\hline & \multicolumn{3}{|c|}{ Differentially regulated miRNA+/- 2 -fold $p<0.05$} & \multicolumn{3}{|c|}{ Differentially regulated miRNA+/- 2-fold $p<0.01$} \\
\hline & DataAssist & StatMiner & Common & DataAssist & StatMiner & Common \\
\hline Controlvs. LVL & 38 & 41 & 21 & 8 & 14 & 8 \\
\hline Controlvs. HVL & 244 & 218 & 191 & 163 & 137 & 118 \\
\hline LVL Vs. HVL & 183 & 182 & 158 & 90 & 99 & 80 \\
\hline
\end{tabular}

Comparative analysis of significantly differentially regulated miRNAs between Statminer (Integromics) and DataAssist (Applied Biosystems). For both analyses, RNU44, RNU48, and ath-miR-159 controls were eliminated from the analysis. For DataAssist, all miRNAs with CT valve of $<40$ were considered. 


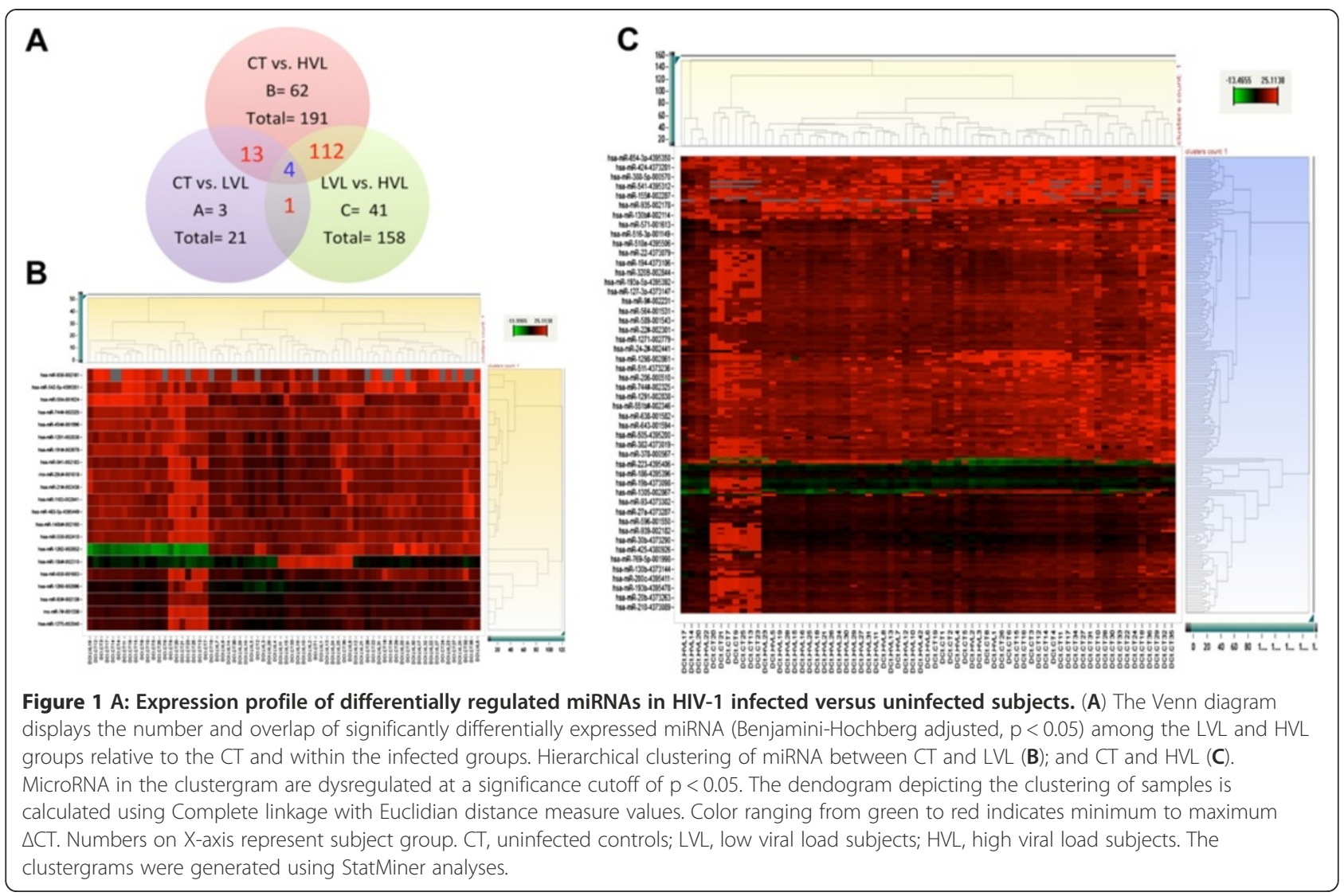

miR-1262 shows downregulation in the CT versus HIV1 positive group, and was upregulated in the HVL group compared to the LVL group. Further analyses within the HVL group based on viral load ranging from 40,000 to 600,000 RNA copies/ml did not show any additive effect. Together, these results suggest that HIV-1 infection has specific regulatory effects on miRNA expression profiles.

To further assess the clustering of subjects within these groups and across the groups, hierarchical clustering was performed in a comparison of the control and LVL groups (Figure 1B) and of the control and HVL groups (Figure $1 \mathrm{C}$ ). When the control and LVL groups are compared, subjects from each group formed several clusters and these clusters dispersed intermittently. Many of the control samples formed a single major cluster, while the remaining control samples were interspersed among the LVL subjects in two other major clusters. However, when we compared the control and HVL groups (Figure 1C), the majority of the subjects from each group clustered distinctly from each other based on their miRNA profile, with an exception of a single separate cluster of HVL containing 4 subjects and control group with 6 subjects from each group. Further analyses of CD4 counts, age and viral load did not show any differences compared to subjects in the major cluster. The comparison of HVL to LVL produced 3 major clusters in LVL and 2 clusters in HVL (Additional file 2: Figure S1). Importantly, these results suggest that the miRNA expression profile within the infected subjects with high viral load is specific. This pattern is different in HIV-1 subjects with undetectable viral load and high CD4 counts, who maintain an expression profile that is similar to uninfected controls. More importantly, this distinct profile is observed in most of the subjects (based on the clustering) and further confirms the specificity.

\section{Validation of miRNA expression by qRT-PCR}

To validate the differentially regulated miRNAs from the microarray results, we randomly selected miRNAs (miRNAs with $>2$-fold change and $\mathrm{p}<0.05$ ) and tested them in independent subjects (using similar selection criteria) by qRT-PCR using miRNA specific Taqman primers and probes (Figure 2A\&B). Results from the comparison of the LVL group and uninfected controls indicate that among the miRNAs tested, six miRNAs (miR-483-5p, miR-16, miR-18b*, miR-376b, miR-938, and miR-1260) exhibit similar fold change pattern as seen in the high throughput results. However, three miRNAs (miR-21*, miR-1262 and miR-1303) showed an opposite fold change pattern to that of the 

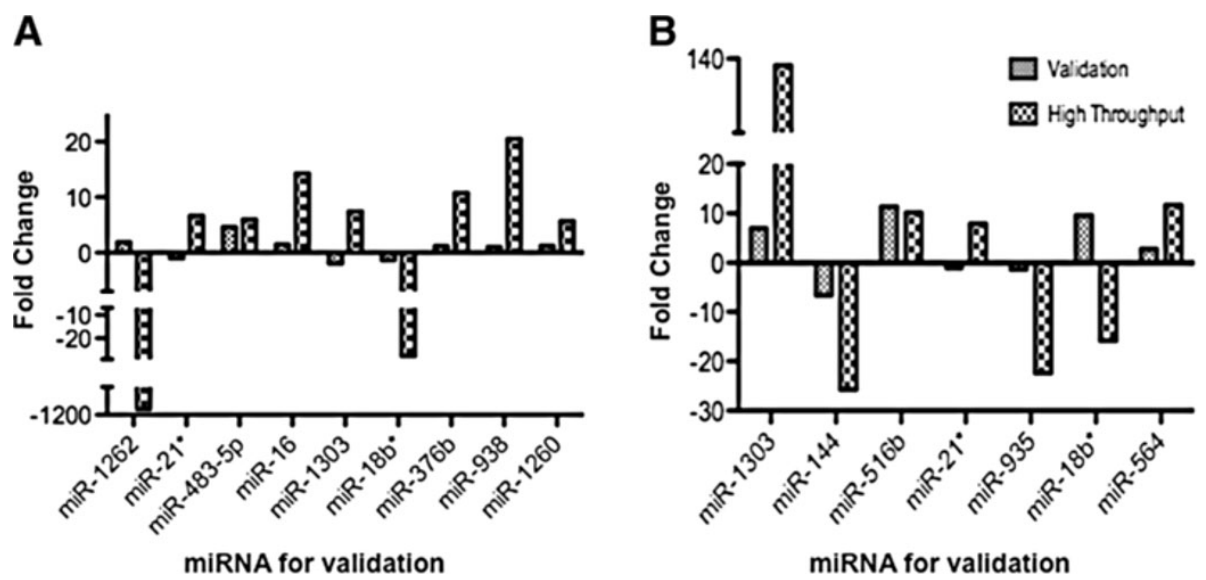

Figure 2 Independent individual validations of randomly selected differentially regulated miRNAs from Taqman array platform. qRTPCR was used to validate the expression of selected miRNAs from the high throughput results (derived by StatMiner) using a specific primer and probe for each miRNA. (A) miRNAs selected from Control versus LVL comparison; (B) miRNAs selected from control versus HVL comparison. Fold increase/decrease was calculated based on normalization to U6. Average fold change for each miRNA represents fold change obtained from independent donors ( $\mathrm{N}=5$ per group).

high throughput results. Specifically, miR-1262 was downregulated and miR-21* and miR-1303 were upregulated in high throughput, while in the qRT PCR, they were upregulated and downregulated, respectively (Figure 2A). Overall these results indicate about $60 \%$ validation (6/9) in terms of similar state of expression (up or down) in both the high throughput array and qRTPCR, however the exact fold change level is different between the two systems as expected.

Similarly, we also tested randomly selected miRNAs in a set of independent HVL subjects relative to uninfected controls (Figure 2B). This comparison indicated that among the seven miRNAs tested, miR-1303, miR-516b and miR-564 showed upregulation in both high throughput array and qRT-PCR, whereas miR-144 and miR-935 showed downregulation in both the array and qRT-PCR assay (Figure 2B). However, two miRNAs (miR-21* and miR-18b*) showed no correlation between the two assays. Further analysis indicates that both miR-21* and miR-18b are low abundant miRNAs and that could be one of the reasons for the observed discrepancies. Overall, validation of miRNA in both sample groups confirms $>65-85 \%$ of the miRNAs suggesting that the array results are reproducible in independent subjects and this effect is more pronounced in the HVL group compared to LVL group. These results are in accordance with the published studies [52-54], indicating that there are differences in differential regulation observed in array versus qRT-PCR assay and 100\% validation is rare if the validation samples are randomly selected as done here. This is further supported when we assessed the significance level ( $\mathrm{p}$ values) and the fold change for these miRNAs (in Additional file 3: Table S2). For instance miR1303 is upregulated in both LVL and HVL subjects in the array, however the significance is $\mathrm{p}=0.024$ in $\mathrm{LVL}$ subjects and $\mathrm{p}=4.67 \mathrm{E}-13$ in HVL subjects. Validation results for miR-1303 by qRT-PCR show similar trend in HVL and opposite pattern in LVL subjects. Additionally, we also observed that five miRNAs that are in concordance with qRT-PCR have highly significant $p$ values $(<1.35 \mathrm{E}-05)$, whereas that of the miRNAs tested in LVL are $<0.05-0.005$. Together these results suggest that differentially regulated miRNAs with significant $\mathrm{p}$ values $(<0.00001)$ has higher validation concordance rate than miRNAs selected based on higher fold change.

\section{HIV-1 induced gene regulation of cellular mRNA transcripts}

MicroRNAs regulate cellular gene expression at the posttranscriptional level, thus silencing and/or downregulating gene expression $[55,56]$. To assess whether a direct correlation exists between the expression patterns of miRNA and mRNA, we assessed the expression profile of mRNA transcripts in PBMCs of the same subjects. Microarray profiling of mRNA samples was normalized with the internal endogenous control and cross-compared between the two groups. Among the 47,000 transcripts tested, 21,852 probes were detected in all samples. Among the total detected probes, $47,11,510$ and 10,007 probes were significantly $(\mathrm{p}<0.01)$ regulated but less than $+/$ - 2 -fold change in control versus LVL, control versus HVL and LVL versus HVL groups, respectively. However, when we narrowed the probes with a fold change of $+/-2$ $(\mathrm{p}<0.01)$, we obtained 0,309 and 182 probes in the 3 groups, respectively (Table 2 ).

Among the 47 probes (41 annotated) differently expressed in the LVL samples compared to CT (FDR corrected with $\mathrm{p}$-value of $<0.01), 36$ were upregulated 
Table 2 Differentially regulated mRNA probes in low and high viral load groups compared to uninfected control group with statistical significance

\begin{tabular}{llllll}
\hline & Detected mRNA probes & $\begin{array}{l}\mathbf{p} \leq \mathbf{0 . 0 1} \\
\text { (All probes) }\end{array}$ & $\begin{array}{l}\mathbf{p} \leq \mathbf{0 . 0 1} \\
\text { (Known probes) }\end{array}$ & $\begin{array}{l}+/-2 \text { fold and } \mathbf{p} \leq \mathbf{0 . 0 1} \\
\text { (All probes) }\end{array}$ & $\begin{array}{l}+/-2 \text { fold and } \mathbf{p} \leq \mathbf{0 . 0 1} \\
\text { (Known probes) }\end{array}$ \\
\hline Control Vs. LVL & 21,852 & 47 & 41 & 0 & 0 \\
Control Vs. HVL & & 11,510 & 9,137 & 309 & 280 \\
LVL Vs. HVL & & 10,007 & 7,820 & 182 & 166 \\
\hline
\end{tabular}

Differentially regulated mRNA probes in within the three study groups based on Illumina HT12 microarray data analyzed with IBD. All probes represent annotated, non-annotated, and curated probes. Known probes represent annotated probes or named genes.

and 11 were downregulated (Table 2). None of the transcripts show more than 1 fold difference $(\mathrm{p}<0.01)$ compared to controls, suggesting that there is not a greater difference between uninfected controls and infected subjects with an undetectable viral load. In contrast, 309 probes were differentially regulated in the high viral load samples compared to the uninfected controls (FDR corrected with a p-value of $<0.01$ with $+/$ - 2 -fold regulation). Of these, 125 were upregulated, and 184 were downregulated. Similarly, we also compared the LVL and HVL groups and identified 182 probes that are differentially regulated. Within these transcripts, a majority (2/3) of them (113) are downregulated and 69 were upregulated. Further, 178 probes were commonly shared between control versus HVL and LVL versus
HVL, whereas none of the mRNAs are shared by all the groups or between control versus LVL group. More importantly, the remaining 131 probes are very specific to the HVL group. Together, these results suggest that subjects with high viral load or AIDS exhibit a unique transcriptome profile compared to the low viral load group. Similar findings were observed in other studies $[4,42,44,45]$. Clustergram analysis of differentially regulated mRNA profiles between control and LVL did not show distinct clustering (Figure 3A), whereas the control versus HVL group comparison showed clusters segregating distinctly, indicating a different transcriptome profile mediated by virus replication within this group (Figure 3B). Similar comparison between LVL and HVL also exhibited a distinct clustering
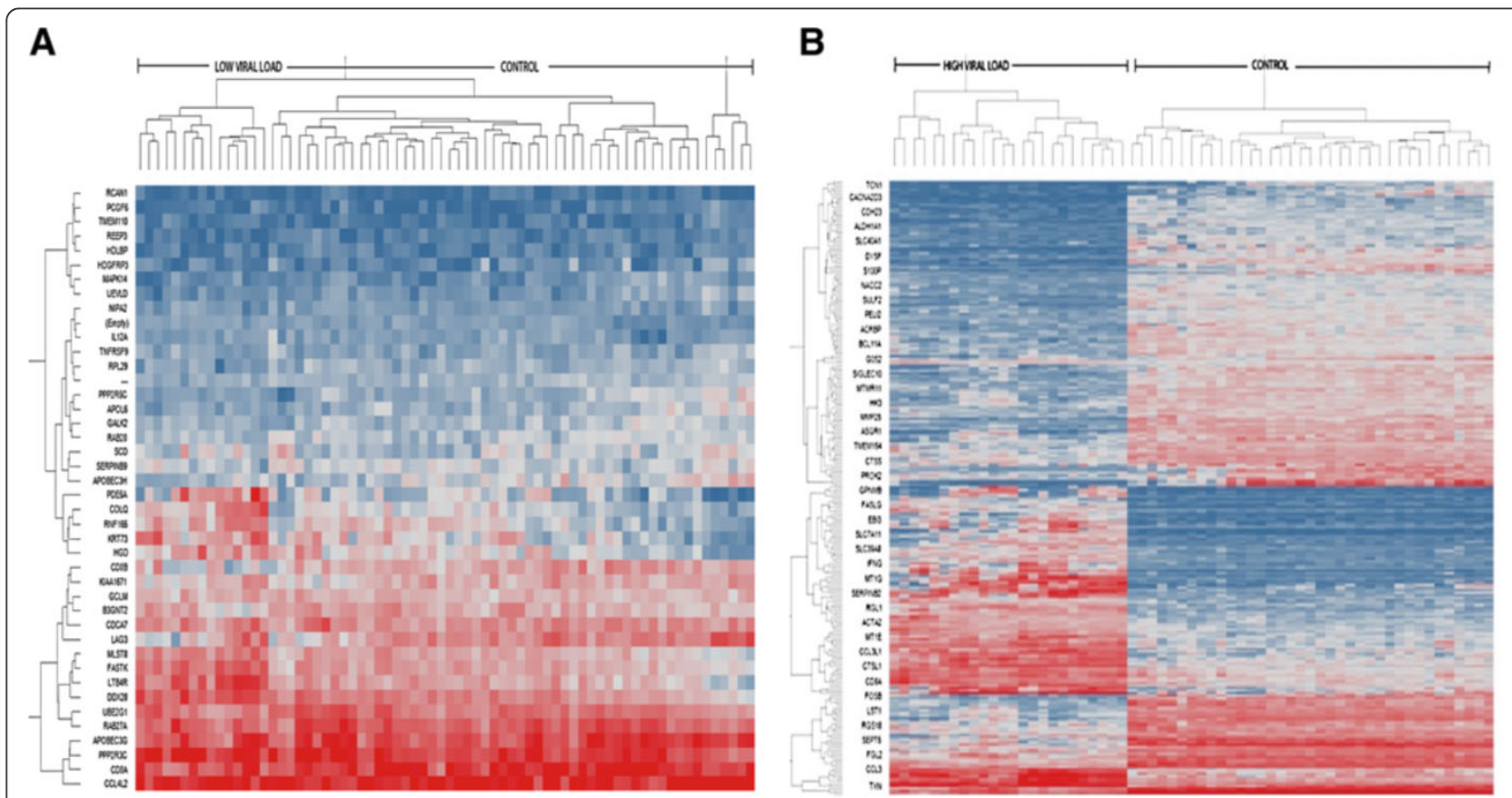

Figure 3 Expression profile of differentially regulated mRNA transcripts in HIV-1 infected versus uninfected subjects. (A). Hierarchical clustering of differentially regulated mRNA in LVL compared to control; and (B), CT and HVL. The probes in this clustergram are significantly differentially regulated $(p<0.05)$. Red indicates high, blue indicates low, and gray stands for no change in level of expression. Numbers on $X$-axis represent subject group; Y-axis represents the gene symbol. CT, uninfected controls; LVL, low viral load subjects; HVL, high viral load subjects. 
pattern (Additional file 4: Figure S2) suggesting that virus replication has significant impact on host cellular gene expression.

\section{Host cellular factors, pathways and biological processes altered by HIV-1}

Next, we analyzed these mRNA transcripts using the STRING and DAVID databases, to identify the biochemical pathways in which they are involved. A comparison of the differentially regulated transcripts in LVL group compared to control group did not show significant changes in cellular pathways, whereas a comparison of the control and HVL groups exhibits distinct pathway regulation (Figure 4A). Though the network shows several interaction groups, four major clusters were apparent; this includes cluster 1 (chemokines and its receptors), cluster 2 (proinflammatory cytokines), cluster 3 (Interferon induced genes) and cluster 4 (metallothionein genes). Among these clusters, the most significantly regulated molecules comprise inflammatory factors, cytokines/chemokine, cell cycle and apoptosis related proteins, cell signaling molecules, factors expressed in response to virus/bacterial infection, innate factors and cell-to-cell interaction molecules. Most importantly, many of the inflammatory factors and cytokines (CXCL5, CCL2, CCL8, CXCL10, CCL7, IL1 $\alpha$, IL-1 $\beta$, IL6 and IL8) are upregulated in the HVL group, whereas antiviral factors and innate immune molecules (members of the defensin family, AIF) were significantly downregulated (Additional file 3: Table S2).

We next assessed the functionality of the significantly dysregulated genes with a focus on those genes that were statistically over represented. An analysis of biological functions, using Gene Ontology Enrichment, shows only four significant functions, including response to virus, IFN $\gamma$ mediated signaling, immune response, and cellular component movement, for the comparison between LVL and controls (Figure 4B). This contrast with the 34
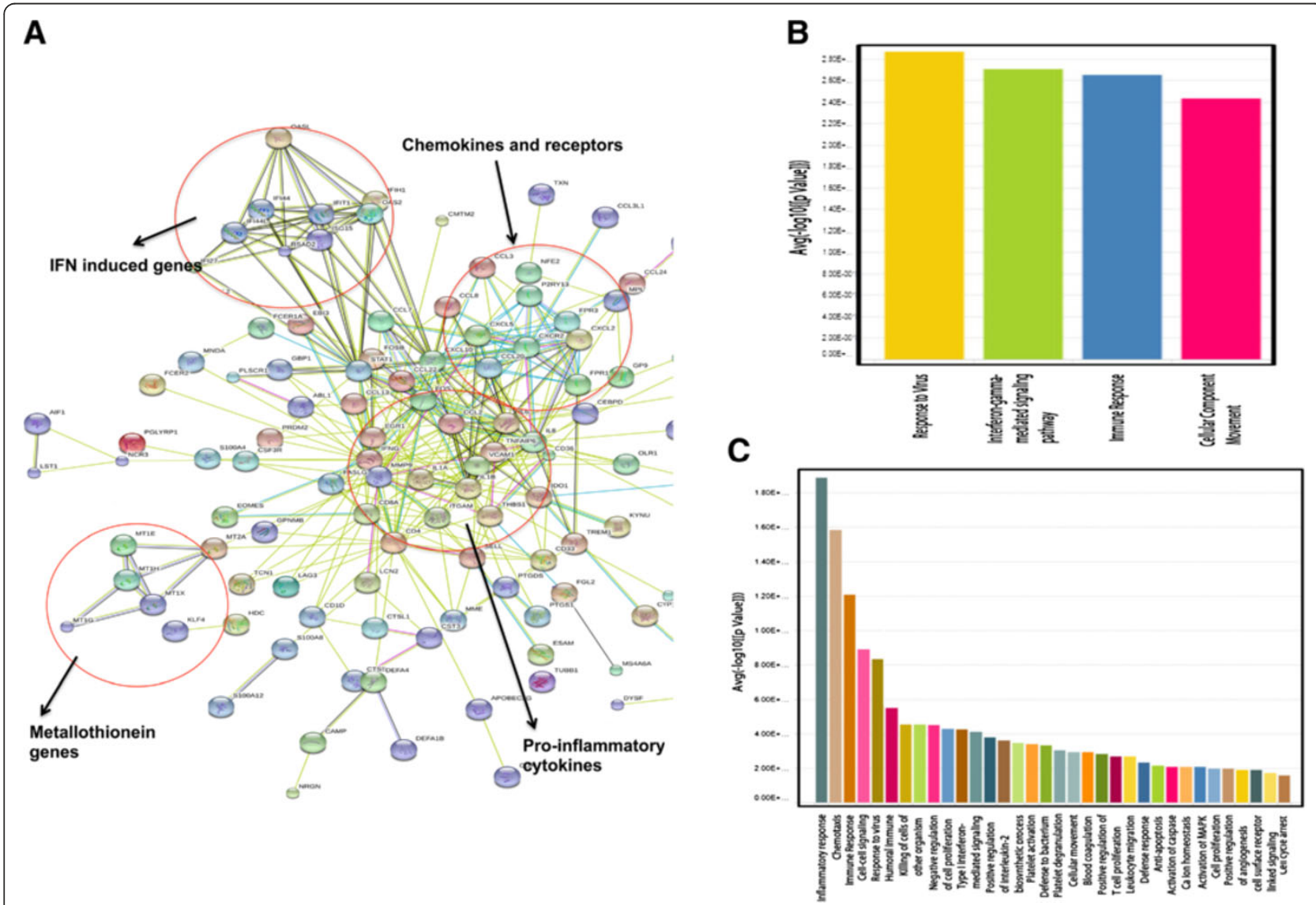

Figure 4 Predicted interaction networks of genes significantly dysregulated in HVL compared to Control. (A) The interactions between genes were identified using STRING software. Colored lines indicate different sources of evidence for each interaction. Circles highlight the predominant clusters within the network. (B \& C) Gene Ontology Enrichment analysis for biological processes using significantly differentially regulated genes in LVL and HVL groups in comparison with $C T$ group. Genes that are differentially regulated in $L V L$ group relative to the $C T$ yielded 4 significant terms for biological processes (B), while those that are differentially regulated in HVL with respect to CT yielded 34 (C). Bar graphs are generated using average -lop of $p$ value for each term on $y$-axis and term name on $x$-axis. $C T$, uninfected control; LVL, low viral load group; HVL, high viral load group. 
significant functions, including inflammatory response, chemotaxis, and immune response, produced from the comparison between HVL and the control group (Figure 4C). These functions relate mostly to inflammatory response, chemotaxis, cell signaling, cell killing and immune regulation. In addition to the gene ontology enrichment, our analyses on host cellular pathways using Ingenuity Pathway Analysis (IPA) indicate several pathways that are distinctly regulated by HIV-1 and high viral load (Additional file 5: Table S3). Differentially regulated RNA transcripts from HVL group target primarily apoptosis and DNA damage pathways (6 out of the top ten pathways) in accordance with the loss of CD4, whereas, LVL did not show similar pathways. Overall, these results suggest that HIV-1 infection resulting in high viral load alters several genes and cellular processes related to HIV-1 induced disease progression.

\section{Computational analyses to identify miRNA-mRNA interactions}

MicroRNA binds to the 3' UTR of the mRNA transcript and regulates gene expression. A single miRNA can potentially regulate single or multiple mRNA and vice versa $[57,58]$. We used GroupMiR [59], a computational method that integrates miRNA and mRNA expression profiles with miRNA target prediction databases (which rely on sequence analysis), to infer interactions between miRNAs and their target mRNAs. This approach of combining expression and sequence analysis has been shown to reduce the number of false positives in miRNA target prediction. These analyses identified 204 miRNAs and 267 mRNAs, plus 572 possible combination pairs with a score ranging from 0.1004 to 0.9832 . The data were used to generate the entire interactome presented in Additional file 6: Figure S3. Further analyses with a few selected pairs indicate that a number of miRNAs (miR-144, miR-376a, miR-503 and miR-935) target a cluster of mRNA including chemokines (CCL2, CCL3, CCL8, IL1 $\beta$, IL6, PLA2G7, DFNA and members of metallothionein gene family), that are part of the inflammatory response (Figure 5). These results suggest that multiple miRNAs could potentially target the same mRNA transcripts with varying strengths (posterior probabilities). For instance, miR-144 and miR-376a exhibit a score of $>0.6$ for the inflammatory gene cluster, whereas, miR-503 and miR-935 exhibit a score of $<0.2$ for the same mRNA transcript, indicating difference in strength of miRNA-mRNA interaction pairs. However, it is important to note that though the strengths are different for these miRNA-mRNA pairs, the inverse

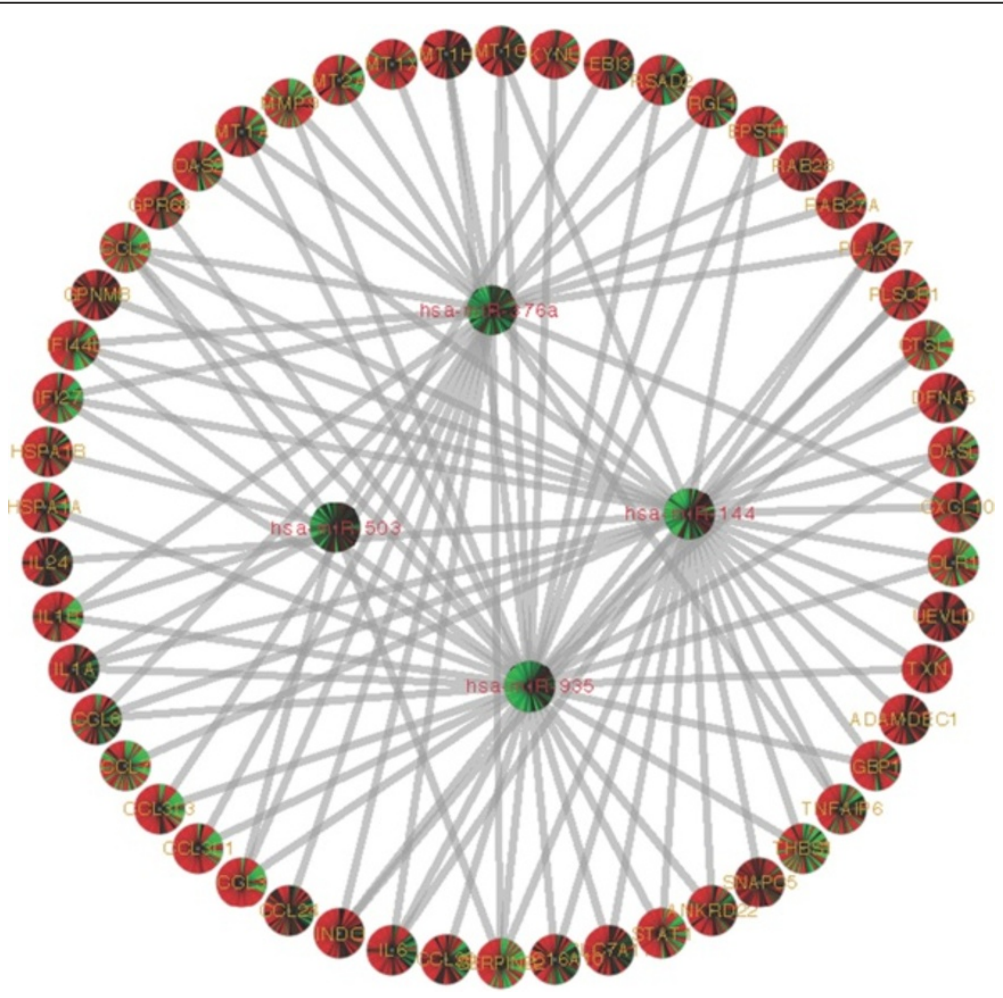

Figure 5 Network of predicted miRNA-mRNA interactions by GroupMiR using results from miRNA and mRNA array and visualized by Cytoscape. Regression-based method was used to predict the potential miRNA (circles) that actively regulate mRNA (squares). Selected miRNA (four) were chosen from a list of miRNAs and their targets. Red and green within the mRNA represents up and down regulation, respectively. Each slice within the circle represents HIV-1 infected subject. 
correlation between the miRNA and mRNA are constant. The four miRNAs shown in Figure 5 are significantly downregulated $(<2$-fold, $\mathrm{p}<0.05)$ in $\mathrm{HVL}$ samples, whereas 47 of the 50 mRNAs are significantly upregulated $(>2$-fold, $\mathrm{p}<0.05)$ in HVL subjects. To further verify and correlate the significance of these interactions, we selected a number of mRNA and miRNA pairs and assessed their expression levels. This includes host cellular genes IL6, NRGN (Neurogranin), AIF1, NCR3, ALDH1A1, CCL2 and CCL8 as well as the predicted respective miRNAs. The results presented in Table 3 were derived by comparing the control subjects to HIV-1 positive subjects (LVL and HVL). First we compared the correlative effects of the miRNA and mRNA in LVL and HVL groups compared to control group. The selected mRNA-miRNA pairs showed a significant inverse correlation in their expression pattern, except for CCL8miR-512-3p and CCL8-miR-518d-3p $(\mathrm{p}>0.05)$ in HVL comparisons. Prediction analyses identified that NRGN could be potentially regulated by 2 miRNA, whereas CCL2 and CCL8 could be potentially regulated by several miRNAs. Conversely, miR-935 has potential binding sites in IL6, CCL2 and CCL8 transcripts in the 3' UTR region. These results highlight the ability of GroupMiR to accurately predict interactions with high levels of variations found in in vivo. However, additional extensive in vitro analyses, beyond the scope of this manuscript, are required to confirm these interactions.

\section{Role of CD4 count and viral load on host cellular transcriptome}

To examine whether other factors, besides the presence of high viral load, are responsible for the differential miRNA and mRNA profiles, we selected a number of cellular factors (CCL2, CXCL5, IL6, IL8 and NRGN) from our predictions and assessed their expression between the three groups. The presence of high viral load significantly influences the expression of CCL2, CCL8, IL6 and IL8, whereas NRGN was inhibited (Figure 6). HIV-1 infection itself down regulated NRGN expression as seen in LVL. Similarly, Defensin and AIF1 were also down regulated by infection and HVL significantly augmented this inhibition (Additional file 3: Table S2). Next, we assessed whether there is a possible correlation with CD4 count, nadir CD4 count, viral load, and host

Table 3 Predicted miRNA-mRNA interacting pairs by GroupMiR in comparison with miRNA profiling studies

\begin{tabular}{|c|c|c|c|c|c|c|c|c|}
\hline \multicolumn{3}{|c|}{ mRNA-miRNA pairs by GroupMiR } & \multicolumn{2}{|c|}{ Control vs LVL (miRNA) } & \multicolumn{2}{|c|}{ Control vs HVL (miRNA) } & \multicolumn{2}{|c|}{ LVL vs HVL (miRNA) } \\
\hline mRNA & miRNA & $P$ value & Fold Change & $P$ value & Fold change & $P$ value & Fold change & $P$ value \\
\hline AlF1 (-2.67) & miR-483-5p & 0.8026 & 5.969 & $0.00410^{* *}$ & 17.088 & $0.000000205^{* *}$ & 2.863 & $0.0125^{*}$ \\
\hline $\operatorname{AlF1}(-2.67)$ & miR-605 & 0.9418 & 1.164 & 0.871 & 29.059 & $0.0000000127^{* *}$ & 24.961 & $0.0000000542^{* *}$ \\
\hline $\operatorname{ALDH1A1}(-2.11)$ & miR-222 & 0.2438 & 1.710 & 0.229 & 2.558 & 0.0611 & 2.856 & $0.000129^{* *}$ \\
\hline $\operatorname{ALDH1A1}(-2.11)$ & miR-155 & 0.7276 & 5.183 & 0.0570 & 16.778 & $0.000103^{* *}$ & 3.237 & $0.000000808^{* *}$ \\
\hline NCR3 $(-2.3)$ & miR-34a & 0.1528 & 1.936 & 0.547 & 20.678 & $0.0000153^{* *}$ & 10.681 & $0.000576^{* *}$ \\
\hline NCR3 $(-2.3)$ & miR-660 & 0.119 & 2.276 & 0.393 & 6.452 & $0.00778^{* *}$ & 2.835 & $0.0233^{*}$ \\
\hline NRGN (-4.06) & miR-210 & 0.9606 & 2.931 & 0.162 & 10.536 & $0.000121^{* *}$ & 3.591 & $0.00276^{* *}$ \\
\hline NRGN (-4.06) & miR-564 & 0.9702 & 2.467 & 0.229 & 11.667 & $0.00000170^{* *}$ & 4.730 & $0.00307^{* *}$ \\
\hline IL6 (4.01) & miR-144 & 0.8496 & -1.181 & 0.855 & -25.7093 & $0.00000508^{* *}$ & -21.786 & $0.000241^{* *}$ \\
\hline IL6 (4.01) & miR-376a & 0.8812 & -1.264 & 0.697 & -2.638 & $0.0185^{*}$ & -2.088 & $0.000000797^{* *}$ \\
\hline IL6 (4.01) & miR-935 & 0.227 & -1.706 & 0.378 & -22.431 & $0.0000000179^{* *}$ & -13.141 & $0.0000135^{* *}$ \\
\hline CCL2 (4.53) & miR-144 & 0.1506 & -1.181 & 0.855 & -25.709 & $0.00000508^{* *}$ & -21.786 & $0.000241^{* *}$ \\
\hline CCL2 (4.53) & miR-196b & 0.1026 & 2.167 & 0.121 & -1.071 & 0.898 & -2.321 & $0.00479^{* *}$ \\
\hline CCL2 (4.53) & miR-376a & 0.1272 & -1.264 & 0.697 & -2.638 & $0.0185^{*}$ & -2.088 & $0.000000797^{* *}$ \\
\hline CCL2 (4.53) & miR-503 & 0.1154 & 1.624 & 0.403 & -3.256 & $0.0480^{*}$ & -5.288 & $0.00788^{* *}$ \\
\hline CCL2 (4.53) & miR-590-3P & 0.1122 & 3.077 & 0.099 & -2.00 & 0.252 & -6.150 & $0.00000113^{* *}$ \\
\hline CCL2 (4.53) & miR-935 & 0.1732 & -1.706 & 0.378 & -22.431 & $0.0000000179^{* *}$ & -13.141 & $0.0000135^{* *}$ \\
\hline CCL8 (5.11) & miR-25 & 0.5172 & -1.095 & 0.878 & 2.893 & $0.00117^{* *}$ & 3.169 & $0.00781^{* *}$ \\
\hline CCL8 (5.11) & miR-376a & 0.119 & -1.264 & 0.697 & -2.638 & $0.0185^{*}$ & -2.088 & $0.000000797^{* *}$ \\
\hline CCL8 (5.11) & miR-512-3p & 0.1184 & -1.508 & 0.609 & 3.942 & $0.0298^{*}$ & 5.950 & $0.00829^{* *}$ \\
\hline CCL8 (5.11) & miR-518d-3p & 0.1302 & -1.124 & 0.892 & 4.867 & $0.00491^{* *}$ & 5.471 & $0.00367^{* *}$ \\
\hline CCL8 (5.11) & miR-935 & 0.4236 & -1.706 & 0.378 & -22.431 & $0.0000000179^{* *}$ & -13.141 & $0.0000135^{* *}$ \\
\hline
\end{tabular}

Fold changes and $p$-values for the miRNA and mRNA in infected PBMC compared to the uninfected controls determined by RealTime StatMiner. ${ }^{*}=p<0.05$. 

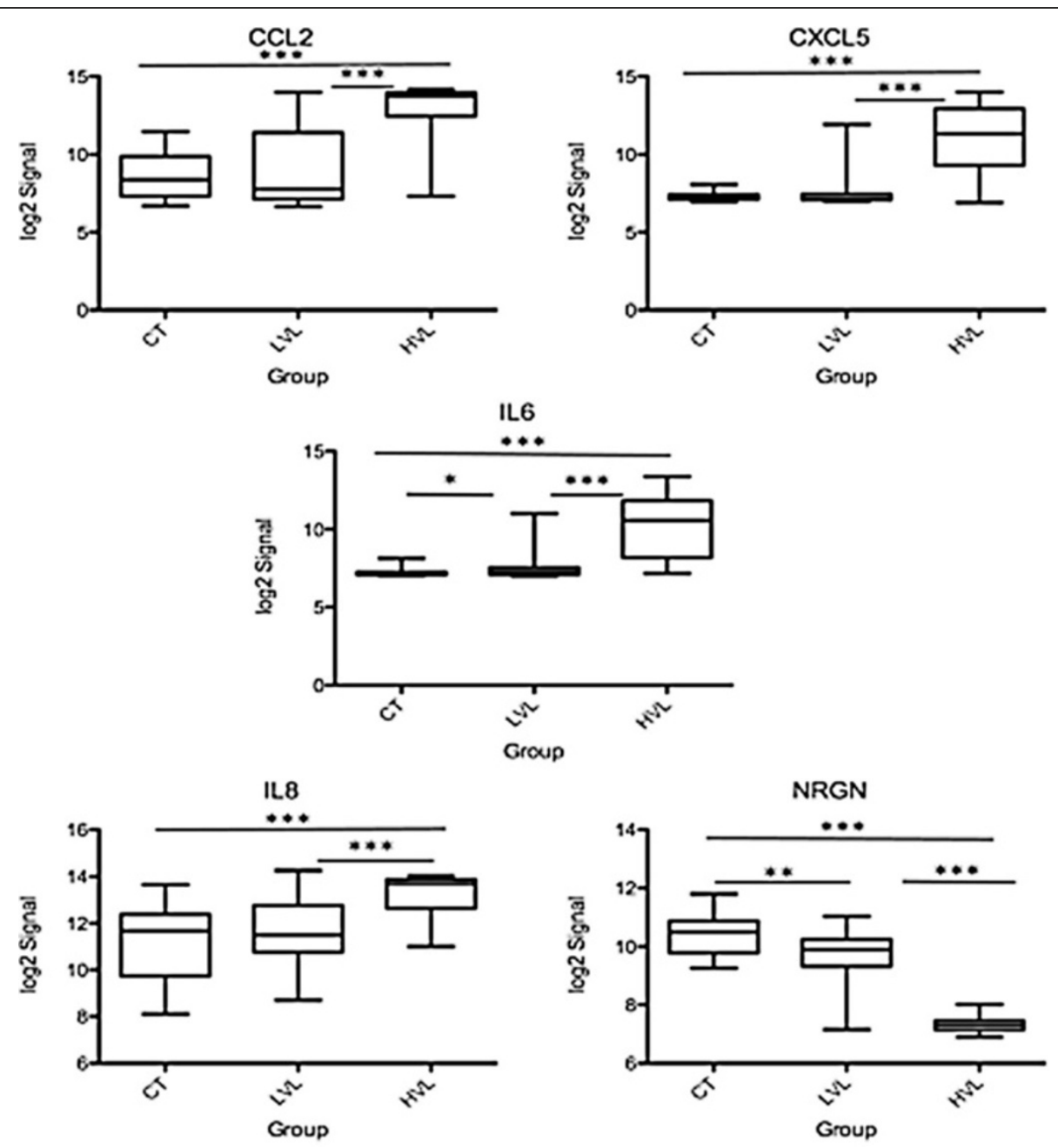

Figure 6 Expression of selected transcripts among the CT, LVL, and HVL groups. Unpaired Student's t-test was used to assess significance between the three groups. $\left(^{*}=p<0.05,{ }^{* *}=p<0.01,{ }^{* *}=p<0.0001\right)$. $C T$, uninfected controls; LVL, low viral load group; HVL, high viral load group.

cellular factors (Table 4). As CD4 count is one of the major determinants of HIV infection and plays a role in altering the transcriptome profile [4], we performed Spearman correlations between the CD4 count, nadir CD4 count, viral load and the signal intensity of each selected transcriptome within the three groups of subjects. None of the transcripts significantly correlate with either CD4 count or nadir CD4 count within the uninfected control group (Table 4; panel A). Within the LVL group, IL6 and NRGN significantly correlate with CD4 count $(\mathrm{p}<0.01$ and $\mathrm{p}<0.05)$ and not with the nadir CD4 count (Table 4; panel B). In the HVL group, IL8 showed a significant correlation with CD4 count $(\mathrm{p}<0.05)$ and nadir CD4 count $(\mathrm{p}<0.001)$, whereas, IL6 correlated with nadir CD4 count only (Table 4; panel C). Next, we assessed, whether the level of viral load within the HVL group is directly or inversely correlated with host cellular factor expression, and results indicate that none of the transcripts significantly correlate with either CD4 count or nadir CD4 count. Together, these results indicate that cellular factors are significantly altered by viral load exceeding 50,000 copies/ml, however the increase in viral copy number $(>500,000 / \mathrm{ml})$ did not alter the expression proportionately suggesting a threshold effect.

\section{Validation of host cellular factors regulated by HIV-1 at RNA and protein level}

Furthermore, we selected a few mRNAs from the differentially regulated mRNA from Additional file 3: Table S2 (AIF, CCL2 and IL6) and validated them in independent HVL donors (Figure 7A). Results indicate that AIF is downregulated in both the array as well as in qRT-PCR assay, whereas CCL2 and IL6 qRT-PCR showed an upregulation, coinciding with the array data. Although the number of transcripts validated is small, these results suggest that validation of mRNA between the array and 
Table 4 A-C: Spearman correlations between selected transcript signals and CD4 count, Nadir CD4 count, and viral load control group

\begin{tabular}{|c|c|c|c|c|c|c|c|}
\hline & & CD4 Count & & Nadir CD4 & & & \\
\hline \multirow[t]{7}{*}{ (A) Control group } & Transcript & Spearman $r$ & $p$-value & Spearman $r$ & $p$-value & & \\
\hline & CCL2 & -0.00296 & 0.986 & 0.156 & 0.358 & & \\
\hline & CXCL5 & 0.0385 & 0.821 & 0.101 & 0.553 & & \\
\hline & IL6 & -0.0236 & 0.877 & 0.0424 & 0.799 & & \\
\hline & IL8 & 0.0234 & 0.891 & 0.227 & 0.176 & & \\
\hline & NRGN & -0.186 & 0.278 & -0.172 & 0.309 & & \\
\hline & & CD4 Count & & Nadir CD4 & & & \\
\hline \multirow[t]{6}{*}{ (B) LVL group } & Transcript & Spearman $r$ & $p$-value & Spearman $r$ & $p$-value & & \\
\hline & CCL2 & -0.281 & 0.133 & -0.193 & 0.283 & & \\
\hline & CXCL5 & 0.025 & 0.895 & -0.026 & 0.883 & & \\
\hline & IL6 & -0.488 & $0.006^{*}$ & -0.185 & 0.304 & & \\
\hline & IL8 & -0.219 & 0.246 & -0.143 & 0.427 & & \\
\hline & NRGN & 0.41 & $0.024^{*}$ & 0.314 & 0.074 & & \\
\hline \multirow[t]{7}{*}{ (C) HVL group } & & CD4 Count & & Nadir CD4 & & Viral load & \\
\hline & Transcript & Spearman $r$ & $p$-value & Spearman $r$ & $p$-value & Spearman $r$ & p-value \\
\hline & CCL2 & -0.022 & 0.918 & -0.084 & 0.695 & 0.202 & 0.355 \\
\hline & CXCL5 & -0.277 & 0.190 & -0.367 & 0.078 & 0.190 & 0.385 \\
\hline & IL6 & -0.324 & 0.122 & -0.414 & $0.044^{*}$ & -0.199 & 0.364 \\
\hline & IL8 & -0.495 & $0.014^{*}$ & -0.609 & $0.001^{* *}$ & -0.085 & 0.670 \\
\hline & NRGN & 0.224 & 0.292 & 0.153 & 0.475 & 0.143 & 0.516 \\
\hline
\end{tabular}

Spearman correlations between selected transcripts and CD4 count, Nadir CD4 count, and viral load (where applicable) within the three study groups obtained from GraphPad Prism software set to produce two-tailed $p$-value. ${ }^{*}=p<0.05 ;{ }^{* *}=p<0.001$.

qRT-PCR assay are concordant with each other specially in transcriptome profiling.

Although, an in vivo validation of serum cytokines and chemokines as well as other markers is ideal, we evaluated the inflammatory factors at the protein level using an in vitro experimental culture model. Normal human primary PBMCs were infected with $\mathrm{HIV}-1$ virus and the levels of cytokines/chemokines released post infection were assessed (Figure 7B). Results indicate that HIV-1 infection significantly increased the expression of inflammatory factors and it is independent of viral tropism. Both NL43 and YU2 significantly upregulated the level of IL6 by 8 -fold, CCL2 by 3 -fold, and CXCL5 by 4 -fold with a significance of $\mathrm{p}<0.05$ in multiple donors. However, the upregulation was about 2.5-fold for CCL8 $(\mathrm{p}>0.05)$ and 8 -fold with $\mathrm{p}<0.05$ for IL8 in NL43 infected PBMCs, whereas it was 3 -fold $(p>0.05)$ in YU2 infected PBMCs. These results exhibit a direct correlation of the mRNA transcript and protein level that showed upregulation in $\mathrm{HIV}-1$ subjects compared to control subjects.

We also tested the expression level of NRGN in these samples by performing qRT PCR. Results indicate that PBMCs infected with NL43 or YU2 showed significant downregulation of the NRGN RNA transcript compared to mock or uninfected control (Figure 7C). To further confirm the expression of NRGN at the protein level, expression of NRGN in cell lysates of PBMCs infected with NL43, YU2 or mock was assessed by immunoblotting using an anti-NRGN antibody (Figure 7D). PBMCs infected with NL43 or YU2 showed significant down regulation of NRGN compared to uninfected or mockinfected control cells. Further quantitative analysis using densitometry scanning indicate that the reduction is $>50 \%$ in PBMCs infected with NL43 virus, whereas a $>80 \%$ reduction was found in PBMCs infected with YU2 virus (Figure 7E). It is not clear why the two different viruses exhibit different levels of down regulation of NRGN. One possible explanation could be due to the infectivity level within these cultures, as both macrophages and CD4+T cells are infected by YU2, whereas, NL43 infects only CD4+ T cells.

\section{Discussion}

Obligatory intracellular pathogens, such as viruses, are known for their ability to manipulate host cells both at the molecular and cellular levels. Studies have shown that the host cellular transcriptome is regulated by HIV1 infection, as part of the cells' response to pathogen insult $[60,61]$. HIV-1 infection results in differential 


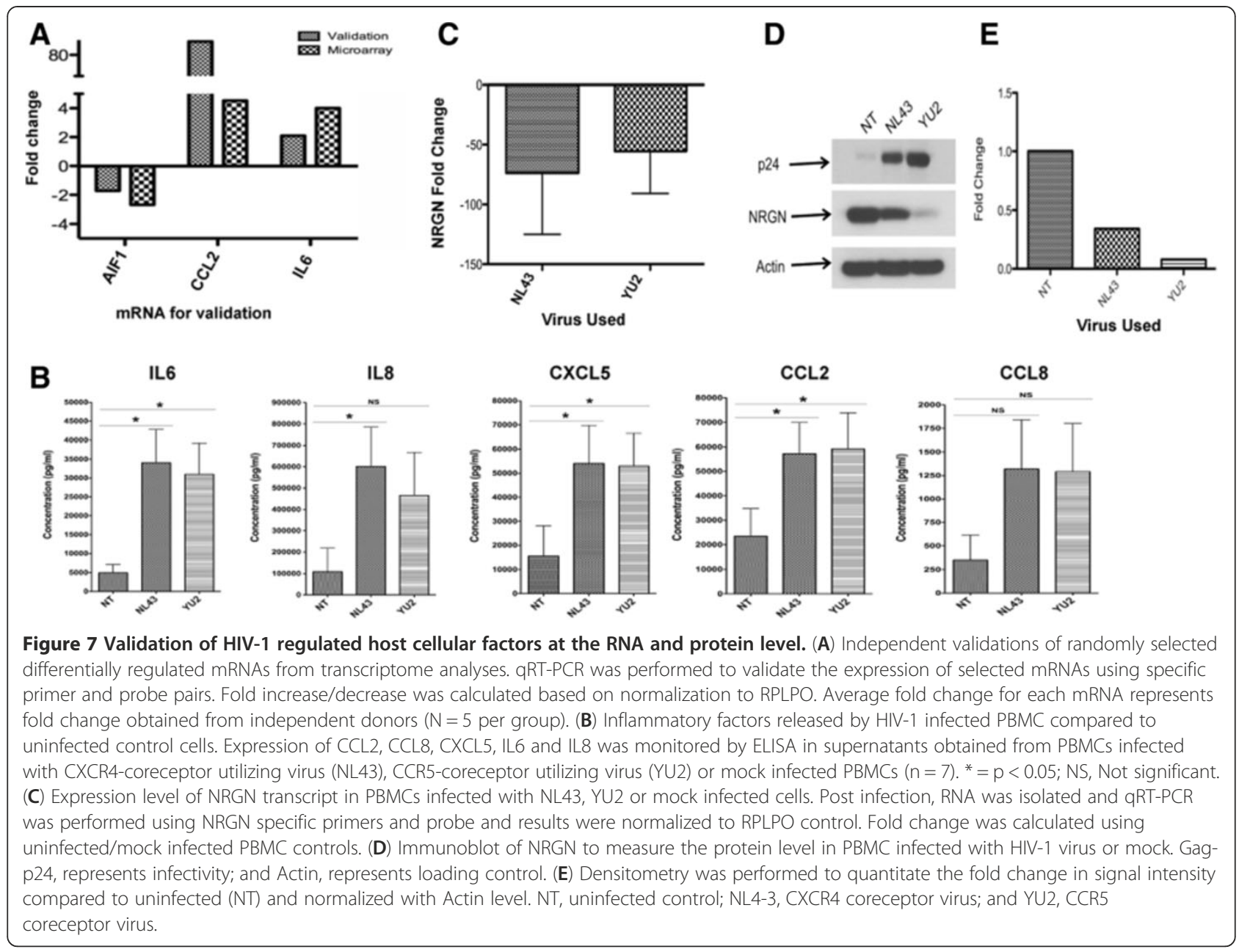

regulation of specific cellular genes in target cells such as CD4+ T cells and monocytes/macrophages suggesting that virus infection alters host cellular proteins either to evade the immune system and/or for optimal viral replication. Considering the prominent role played by miRNA in the regulation of gene expression, we hypothesized that analysis of miRNA in samples from HIV-1 infected individuals may provide information about the molecular network and correlate with the disease status in the host. The novel features of our present study are the following: (i) We have utilized samples from HIV-1 infected hosts characterized for viral load (HVL and LVL), together with appropriate age matched controls; (ii) The samples were derived from a large number of HIV-1 infected subjects; (iii) ours is one of the first in vivo studies to compare miRNA and mRNA simultaneously in PBMCs isolated from a cohort of HIV-1 infected and uninfected subjects. Our results show that HIV-1 regulates the cellular transcriptome significantly through miRNAs, resulting in an inverse correlation of miRNA-mRNA expression. Furthermore, we have also established that there is an interrelationship between multiple miRNA and mRNA interactions and vice versa within the infected individuals.

The majority of miRNAs are transcribed from noncoding DNA, however a number of miRNAs are also known to be expressed along with mRNA suggesting a specific role for mRNA regulation [62-64]. Our comparative analysis of miRNA and mRNA indicate that HIV-1 infection alters both miRNA and mRNA expression profiles in HIV-1 infected subjects compared to uninfected controls. However, the dysregulation of both the RNA species is less prominent in LVL subjects compared to the HVL subjects indicating that viral load has a significant effect. Differentially expressed miRNA between groups indicates both overlapping and group specific miRNA expression. For instance, HIV-1 infection independent of viral load altered expression of only 4 miRNAs, whereas 191 miRNAs are significantly regulated in the high viral load group compared to uninfected controls. Previous studies using PBMCs or CD4+ T cells from infected viremic, elite controllers and multiply exposed 
uninfected (MEU) subjects indicate that HIV-1 infection and/or virus exposure resulted in signature miRNA expression profile further supports the role of HIV-1 induced miRNA regulation $[41,46,48]$.

Although a number of commonly dysregulated miRNAs were noted in these studies, there is also a greater degree of variation between these studies including the current study $[46,48]$. The differences observed between these studies could be due to a number of reasons including array platforms, the patient population, sample size, software used and type of analyses, cut-off set by each group and selection criteria of study subjects. Our study has a large sample size ( $\mathrm{n}=30$ or more) and all of our LVL donors were receiving Highly Active Antiretroviral Therapy (HAART) and had no or undetectable viral load for more than 5 years prior to sample collection. Another possible explanation is the cell types used in these studies (total PBMC vs. purified CD4+ T cells), as these are known to exhibit distinct cell specific miRNA profiles $[65,66]$. It is also important to point out that our HVL group has very low CD4 $+\mathrm{T}$ cell count $(<200$ cells), suggesting that the differentially regulated miRNAs could be the result of non CD4+ T cell types in response to HIV-1 infection.

Results from our analyses identified several miRNA that are uniquely regulated by HIV-1; however the functional consequences of many of these miRNAs are not fully understood. Further analyses on cell specific miRNAs expressed during virus replication suggest a role for miR-132, -125b, -155, -150, -223-382 and Let-7 in CD4+ T cells [67-69] and miR-198, -28-3P, 125b, -198, -223 and -382 in monocytes/macrophages [40,70,71], respectively. In our analyses using PBMCs, we noted differential regulation of miR-132, Let-7, -28-3P, -125b, -223, -382, but no difference in others. Additionally, miRNAs (miR-214, -223, 132, -128, -150, -32, 24, -93, -198, -296, 351 and -17-92 cluster) are known to target viral RNA and possess antiviral activity and innate immune functions [72,73]. Among these miRNAs, miR-214 and miR-223 are significantly down regulated in HVL subjects, whereas miR-32, miR-198, miR-128, miR-150 and miR-15 did not show any change. MiR-146a has been linked to innate immunity and immune activation [74,75] and miR-155 is involved in $\mathrm{T}$ cell proliferation and Treg regulation $[76,77]$. These miRNAs are significantly upregulated in HVL subjects compared to seronegative and LVL subjects, suggesting that inflammatory factors are highly upregulated in subjects with HVL supporting hyper activation of immune cells during AIDS. Our studies have shown that the LVL group is similar to the uninfected controls at the mRNA level as evident in few gene interactions in the STRING pathway analysis of the mRNA array data similar to previous studies [42]. On the other hand, several host cellular pathways and networks including cytokine and chemokine interactions, signaling pathways, IFN induced genes, metallothionein genes and pro-inflammatory cytokines were dysregulated in high viral load in comparison to uninfected controls and low viral load samples. Although we have highlighted immune cell related functions in this analyses further analyses indicate that additional pathways such as apoptosis and cell death are that are distinctly regulated in HVL subjects compared to LVL and control further supporting the CD4 loss observed in this group.

Overall, we found only 41 significantly dysregulated mRNAs between uninfected and LVL and all of these mRNAs had a fold change of less than 2. Meanwhile, the comparisons of CT vs. HVL and LVL vs. HVL yielded 309 and 182 significantly regulated probes respectively. Several of these pathways (IFN signaling, innate response, defensins, cytokine/chemokine signaling and $\mathrm{T}$ cell signaling) that are dysregulated in the high viral load (compared to low viral load), are consistent with the results of other studies using HIV-1 specific target cell types $[4,42-44,78]$. We also noticed APOBEC3G, a cellular defense factor against retroviruses, to be upregulated in the presence of infection (CT vs. LVL) and high viral load (CT vs. HVL), which parallels Rotger et al. and their finding that the gene is upregulated with increasing viral load [45]. Although the current study utilized total PBMC for miRNA and mRNA profiling, the results from this analysis reflect both the direct and indirect effect of HIV-1 on miRNA and mRNA expression as well as capturing the dysregulated genes from both monocyte and $\mathrm{T}$ cell lineages. As it is well established that less than 1\% of the cells in total PBMCs are productively infected by the virus, the significant changes observed here may not be result of a direct effect of HIV-1 infection, but may instead be indirect effects. These indirect effects could be due to nonproductively infected cells, exposure of virus particles (both defective and replication competent) to target and non-target cells as well as cellular and viral factors released from the infected cells that could affect bystander cell population. Our results show that it would certainly be worthwhile to perform cell specific analysis on HIV-1 target cells using a focused array to assess the effect that infection on the RNA species within the individual cell types, but this is beyond the scope of this manuscript.

To study the interaction between the miRNA and mRNA, we chose not to simply rely on online interaction databases, instead, we elected to use our array data as a supplement to GroupMiR. Notably, our analysis has predicted pathway-associated targets for many miRNAs, suggesting that miRNAs effectively target host cellular pathways during infection and disease progression. Although several of the predicted miRNA-mRNA 
pairs showed inverse correlation, we also observed a few positive correlations in mRNA regulation. Specifically, in case of inflammatory factors, IL6, CCL2 and CCL8 are inversely regulated by miR-144, miR-376a, miR-935 and miR-146a, whereas, in our study both expression of miR-512-3p-CCL8 and miR-518d-3p-CCL8 are positively correlated in HVL subjects compared to uninfected controls. Most importantly, our studies have identified several new miRNAs that could potentially regulate RNA transcripts such as AIF1, ALDH1A1, NCR3 and NRGN that warrant further research. Though it is established that the host transcriptome is regulated by HIV-1, it is not clear how transcription is regulated. Based on our results, it is possible to predict that one of the mechanisms that the virus employs is the use of miRNA, as regulation of a single miRNA could modulate multiple mRNAs that belong to specific pathways, which would be beneficial for the virus.

The presence of high viral load has an impact on several mRNA transcripts, however, the effect of other factors such as CD4 count and nadir CD4 has a significant negative correlation for IL8; this is consistent with data in the literature that associates infection with elevated IL8 [79]. This was not surprising because the nadir CD4 counts for some of the HVL subjects were quite low. Furthermore, this paralleled Stone et al.'s findings of elevated IL6 in HIV patients after an immune restoration [80]. We also found significant correlations within LVL for IL6 and NRGN and CD4. Interestingly, we found no significant correlations with the level of viral load, indicating that the presence of virus has an effect on producing differential mRNA expression but the level of the viral load makes no additional difference. Together these observations suggest that HIV-1 infection, irrespective of its severity, has significant impact on host cellular transcripts and gene expression resulting in immune dysregulation. These findings might provide further explanation on why immune function is never restored back to normal in HAART treated HIV-1 subjects with no detectable virus load. Our study of miRNA and mRNA profiles in individuals infected with low and high levels of viral load has significant clinical relevance. The finding of several miRNAs that are specific to a particular viral load could lead to the use of these miRNAs as biomarkers for the determination of prognosis of the disease.

\section{Conclusions}

In this study, we have shown that the expression profile of miRNA and mRNA in HIV-1 infected individuals with high viral load is very distinct from controls indicating that gene expression is significantly altered in HIV-1 target cells in response to virus replication. We also identified several novel genes whose expression levels vary significantly in our cohorts, and which have potential as therapeutic targets. By using a comparative profiling of miRNA and mRNA simultaneously in these study populations we are able to predict putative miRNA-mRNA interactions and a mathematical model to explain the expression of a given mRNA target in terms of its interaction with the miRNAs thought to regulate its expression. Importantly, we identified several new mRNA targets (Defensin, Neurogranin, AIF) as well as the miRNAs that could be involved in regulating their expression through the miRNA-mRNA interaction. These findings will open new avenues to target host cellular factors using RNAi and anti-miRNA technologies to combat HIV-1.

\section{Methods}

\section{Study Population and selection criteria}

All donor samples were obtained from the Pittsburgh site of the Multicenter AIDS Cohort Study. The study was approved by the Institutional Review Board (IRB) at the University of Pittsburgh and samples were collected using informed consent forms according to the University policies. Donor blood was processed by Ficolhypaque and PBMCs were stored in RNALater or frozen in $\mathrm{LN}_{2}$ for further use. The study population comprised uninfected seronegative controls $(\mathrm{N}=36), \mathrm{HIV}-1$ positive subjects with low viral load $(<40$ copies $/ \mathrm{ml})(\mathrm{N}=32)$, and HIV-1 positive subjects with a high viral load $(>50,000$ copies $/ \mathrm{ml})(\mathrm{N}=31)$ at the time of sample collection. Details of the subjects including age, CD4 counts, nadir CD4 counts, viral load at the time of sample collection, viral load for the past 5 years and number of years in ART/HAART are included in Table 5.

\section{MicroRNA profiling and data analysis}

Total PBMCs were used for RNA isolation. Total RNA was isolated using the MirVANA kit (Applied Biosystems), as suggested by the manufacturer. RNA quality was determined using the Nanodrop2000 spectrophotometer and Bioanalyzer and samples with high RNA quality (RIN values between 8 and 9.7) were used for further profiling. The human microRNA microfluidic card set v3.0 (Applied Biosystems) was used for miRNA profiling of samples. This set enables quantitation of 768 miRNA in total, which include 754 targets, 4 endogenous controls (MammuU6/U6 snRNA run in quadruplicates, and RNU44, RNU48, and ath-miR-159a run in duplicates). One $\mu \mathrm{g}$ of RNA was reverse transcribed using *Taqman microRNA reverse transcription kit (Applied Biosystems, CA) along with megaplex primer pools, human pools set v3.0; the resulting PCR product was loaded on to array cards with Taqman Universal Master Mix II-No UNG, which were run on "ViiA7 Real-Time PCR system according to manufacturer's 
Table 5 Clinical status, viral load, CD4 counts of subjects used in this study

\begin{tabular}{llll}
\hline Parameters & Control HIV-1 negative & HIV-LVL (HIV-1- low viral load) & HIV-HVL (HIV-1 high viral load) \\
\hline Number per group & 36 & 32 & 31 \\
Years post SC & N/A & $6-26$ & $0-11$ \\
Age median (range) & $50.5(28-74)$ & $51(34-62)$ & $42(28-65)$ \\
CD4 count range (time of sample) & $385-2194$ & $193-1421$ & $38-378$ \\
Nadir CD4 median (range) & $562(240-1074)$ & $259.5(13-639)$ & $188(29-469)$ \\
Viral load (time of sample collection) & N/A & $<40$ & $46,053-561,627$ \\
Viral load variations for past 5 years & N/A & Remain $<40$ & High \\
Years on HAART/ART & N/A & $2-14$ & $0-7$ \\
\hline
\end{tabular}

protocol as well as standardized protocols developed in our laboratory.

Initial miRNA expression data was analyzed using integrated ViiA7 software (Applied Biosystems). Each run was exported individually using the auto threshold and was then uploaded to RealTime StatMiner software (Integromics, PA) for further analysis. Detectability threshold for miRNA assays was set to $\mathrm{Ct}$ value less than or equal to 36 (as no preamplification step was included) in more than $25 \%$ of all samples in each group. We used Grubbs' method to eliminate outliers within technical replicates and imputed missing $\mathrm{Ct}$ values based on an aggregation to the median. We used the Genorm method to select the endogenous control U6-snRNA/ MammU6 for normalization based on stability scoring across the samples in each group. The aggregation method selected was to the median. Differentially expressed miRNA between different groups were identified using parametric $t$-test or LIMMA (one factor analysis) and were then sorted using the BenjaminiHochberg false discovery rate (FDR) method with adjusted $\mathrm{p}$-values $<0.05$ and $\mathrm{p}<0.01$. The fold changes were obtained using linear RQ values. Hierarchical clustering for differentially expressed miRNA was performed with their corresponding $\mathrm{dCt}$ values across the samples in different groups with 'Complete linkage' clustering method and 'Euclidean' distance measure for dendrograms.

\section{mRNA profiling and data analysis}

For whole genome transcriptome analysis, we used Illumina HT-12 V4 array bead chips (Illumina, Inc., San Diego, CA, USA) for mRNA profiling of the different groups (Control, LVL and HVL) in the study. Each array targets about 47,231 probes that include 28,688 wellcharacterized or annotated coding transcripts along with 11,121 coding transcripts with provisional annotation and remaining being non-coding transcripts and splice variants. RNA samples $(1 \mu \mathrm{g})$ were labeled using the 'TotalPrep RNA' labeling kit (Ambion), reverse transcribed to cDNA, hybridized onto array bead chips overnight on rocker and scanned on 'iScan system' according to the manufacturer's protocols as well as standardized protocols developed by the Genomics and Proteomics Core Laboratories at the University of Pittsburgh. Datasets will be deposited in NCBI gene expression and hybridization array data repository GEO database.

\section{Statistical analysis}

Data analysis was performed using the Illumina BeadStudio software to delineate the false discovery rate (FDR) and differences with statistical significance $(\mathrm{p}<0.05)$. Initial raw data analysis and cubic spline normalization was done using the BeadStudio Gene expression module (Illumina, Inc.). The normalized sample probe profile and control probe profile were then uploaded to Integrated Biomarker Discovery (IBD) (Integromics) for further data analysis. A total of 21,852 probe sets were detected in all samples of different groups (Control, LVL and HVL) with present call filter set to probes detected in $>75 \%$ of samples in each group. Linear models for microarray data (LIMMA) (one factor analysis) was used to identify differentially expressed genes/probes between different groups. These genes were further sorted based on the Benjamini-Hochberg false discovery rate (FDR) method with an adjusted p-value $<0.05$ and fold change cut-off of at least 2-fold up/down regulation. Hierarchical clustering for differentially expressed genes/probes was performed using their corresponding detection signal values across the samples in different groups with 'Complete linkage' clustering method and 'Euclidean' similarity measure for dendrograms. Statistical analyses for in vitro experiments and figures were generated using GraphPad Prism.

\section{Validation of differentially regulated miRNA and mRNA}

Based on the data analyses, selected miRNA and mRNA were verified by qRT-PCR. RNA samples from independent subjects $(n=5)$ were used to validate the high throughput microarray results using miRNA and mRNA specific primers and probes as suggested by the manufacturer 
(Applied Biosystems). Following normalization to U6 (miRNA) or RPLPO (mRNA), the data were exported and analyzed in RealTime Statminer software, as suggested by the manufacturer.

\section{Pathway analysis}

To determine gene interactions and correlation networks, we used IPA and STRING. The Gene Ontology Enrichment (GO) analysis tool within IBD was used to detect biological annotations that are statistically overrepresented in the list of differentially regulated genes/ probes between different groups (e.g. Control and LVL). With minimum number of overlapping genes equal to 3 and FDR threshold of $<0.05$, both singular and concurrent enrichment analysis were carried out. Significant terms for biological processes (BP), molecular functions (MF) and cellular components (CC) were retrieved, arranged in an order based on p-value for corresponding term.

\section{Interaction of miRNA-mRNA using GroupMiR}

We used GroupMiR [59], a computational method that integrates miRNA and mRNA expression profiles with miRNA target prediction databases (which rely on sequence analysis), to infer interactions between miRNAs and their target mRNAs. This approach of combining expression and sequence analysis has been shown to reduce the number of false positives in miRNA target prediction. We downloaded the predictions from MicroCosm database (http://www.ebi.ac.uk/enright-srv/micro$\operatorname{cosm} / \mathrm{htdocs} / \operatorname{targets} / \mathrm{v} 5 /$ ) and used these as priors for our method. We used the default parameters of GroupMiR for this analysis [59]. The analysis was done with HVL and LVL samples where paired miRNA and mRNA expression profiles are available. The $\log 10$ ratios of the mRNA expression levels of HVL/LVL samples to the average values of control samples were used. For miRNAs, the $\log 10$ ratios of the average values of control samples to the dCT values of HVL/LVL samples were used.

\section{Biological validation of HIV-1 regulated factors in vitro}

HIV-1 induced cytokines and chemokines were tested in an in vitro model by infecting normal donor blood derived PBMCs infected with CXCR4 - or CCR5 coreceptor utilizing viruses (NL43 and YU2 respectively) as described [81,82]. Infection was confirmed via a HIV-1 p24 ELISA. Seven days post infection, the supernatant was assessed for the production of CCL2, CCL8, IL6 and IL8 by ELISA (BD Biosciences, CA) and RNA and cell lysates from infected cells was assessed for NRGN by qRT-PCR and western blot, respectively [83].

\section{Additional files} Additional file 1: Table S1. Differentially regulated miRNA in low and
high viral load groups compared to uninfected control group using.

Additional file 2: Figure S1. Hierarchical clustering of miRNAs in HIV-1 infected low viral load (LVL) versus high viral load (HVL) subjects. The dendogram depicting the clustering of samples is calculated using Complete linkage with Euclidian distance measure values. Color ranging from green to red indicates minimum to maximum dCT. Numbers on Xaxis represent subject group. The clustergrams were generated using data from StatMiner analyses.

Additional file 3: Table S2. Differentially regulated mRNA probes in low (LVL) and high viral load (HVL) groups compared to uninfected control group with statistical significance.

Additional file 4: Figure S2. Hierarchical clustering of differentially regulated $m R N A$ in low viral load $(L V L)$ ompared to high viral load $(H V L)$ subjects. The probes in this clustergram are significantly differentially regulated $(p<0.05)$. Red indicates high, blue indicates low, and gray stands for no change in level of expression. Numbers on $X$-axis represent subject group; Y-axis represents the gene symbol.

Additional file 5: Table S3. a: Top ten Canonical pathways representing mRNA that are differentially expressed in LVL group compared to uninfected seronegative subjects. b: Top ten Canonical pathways representing mRNA that are differentially expressed in $\mathrm{HVL}$ group compared to uninfected seronegative subjects.

Additional file 6: Figure S3. Network of predicted miRNA-mRNA interactions by GroupMiR using results from miRNA and mRNA array and visualized by Cytoscape. Regression-based method was used to predict the potential miRNA (circles) that actively regulate mRNA (squares). Differentially regulated miRNA and mRNA in HIV-1 infected subjects compared to the uninfected controls were used to predict the miRNAmRNA pairs. Red and green within the mRNA represents up and down regulation, respectively. Each slice within the circle represents HIV-1 infected subject.

\section{Competing interests}

The authors declare that they have no competing interests.

\section{Authors' contributions}

KD, PN and PI performed miRNA and mRNA arrays and analyzed the data; $K D, P N, H L, A T$ and ZB performed the bioinformatics analyses; VA, JM and KD wrote the manuscript; CR critically revised the manuscript; PN and WB coordinated the study; All authors read and approved the final manuscript.

\section{Acknowledgments}

The authors are grateful to the volunteers and the staff of the Multicenter AIDS Cohort Study for the time and effort that they contributed towards the successful completion of this project. This work was supported by U01 Al35041 based ARRA supplements to VA, JM and CR from NIAID/NIH.

\section{Author details}

${ }^{1}$ Department of Infectious Diseases and Microbiology, Graduate School of Public Health, University of Pittsburgh, 425 Parran Hall, 130 Desoto Street, Pittsburgh, PA 15261, USA. ²Department of Machine Learning, Carnegie Mellon University, Pittsburgh, PA, USA. ${ }^{3}$ Human Genetics, Genome Institute of Singapore, 60 Biopolis Street 02-01, Singapore, Singapore.

Received: 25 October 2012 Accepted: 16 May 2013

Published: 30 May 2013

\section{References}

1. Fellay J, Shianna KV, Telenti A, Goldstein DB: Host genetics and HIV-1: the final phase? PLoS Pathog 2010, 6(10):e1001033.

2. Nowak MA: Variability of HIV infections. J Theor Biol 1992, 155(1):1-20.

3. Salgado M, Lopez-Romero P, Callejas S, Lopez M, Labarga P, Dopazo A, Soriano $V$, Rodes B: Characterization of host genetic expression patterns in HIV-infected individuals with divergent disease progression. Virology 2011, 411(1):103-112. 
4. Wu JQ, Dwyer DE, Dyer WB, Yang YH, Wang B, Saksena NK: Genome-wide analysis of primary CD4+ and CD8+ T cell transcriptomes shows evidence for a network of enriched pathways associated with HIV disease. Retrovirology 2011, 8:18.

5. Vigneault F, Woods M, Buzon MJ, Li C, Pereyra F, Crosby SD, Rychert J, Church G, Martinez-Picado J, Rosenberg ES, et al: Transcriptional profiling of CD4 T cells identifies distinct subgroups of HIV-1 elite controllers. J Virol 2010, 85(6):3015-3019.

6. Li Q, Smith AJ, Schacker TW, Carlis JV, Duan L, Reilly CS, Haase AT: Microarray analysis of lymphatic tissue reveals stage-specific, gene expression signatures in HIV-1 infection. J Immunol 2009, 183(3):1975-1982

7. Wu JQ, Dwyer DE, Dyer WB, Yang YH, Wang B, Saksena NK: Transcriptional profiles in CD8+ T cells from HIV + progressors on HAART are characterized by coordinated up-regulation of oxidative phosphorylation enzymes and interferon responses. Virology 2008, 380(1):124-135.

8. Wout AB V 't, Lehrman GK, Mikheeva SA, O'Keeffe GC, Katze MG, Bumgarner RE, Geiss GK, Mullins Jl: Cellular gene expression upon human immunodeficiency virus type 1 infection of CD4(+)-T-cell lines. J Virol 2003, 77(2):1392-1402.

9. Garcia-Arriaza J, Najera JL, Gomez CE, Sorzano CO, Esteban M: Immunogenic profiling in mice of a HIV/AIDS vaccine candidate (MVA-B) expressing four HIV-1 antigens and potentiation by specific gene deletions. PLoS One 2010, 5(8):e12395.

10. Wout AB V 't, Swain JV, Schindler M, Rao U, Pathmajeyan MS, Mullins Jl, Kirchhoff F: Nef induces multiple genes involved in cholesterol synthesis and uptake in human immunodeficiency virus type 1-infected T cells. J Virol 2005, 79(15):10053-10058.

11. Zaunders J, Dyer WB, Churchill M: The Sydney Blood Bank Cohort: implications for viral fitness as a cause of elite control. Curr Opin HIV AIDS 2011, 6(3):151-156

12. Herbeck JT, Gottlieb GS, Winkler CA, Nelson GW, An P, Maust BS, Wong KG, Troyer JL, Goedert JJ, Kessing BD, et al: Multistage genomewide association study identifies a locus at 1q41 associated with rate of HIV-1 disease progression to clinical AIDS. J Infect Dis 2010, 201(4):618-626.

13. McMichael AJ, Jones EY: Genetics. First-class control of HIV-1. Science 2010, 330(6010):1488-1490.

14. Limou S, Le Clerc S, Coulonges C, Carpentier W, Dina C, Delaneau O, Labib T, Taing L, Sladek R, Deveau C, et al: Genomewide association study of an AIDS-nonprogression cohort emphasizes the role played by HLA genes (ANRS Genomewide Association Study 02). J Infect Dis 2009, 199(3):419-426.

15. Smith MW, Dean M, Carrington M, Winkler C, Huttley GA, Lomb DA, Goedert JJ, O'Brien TR, Jacobson LP, Kaslow R, et al: Contrasting genetic influence of CCR2 and CCR5 variants on HIV-1 infection and disease progression. Hemophilia Growth and Development Study (HGDS), Multicenter AIDS Cohort Study (MACS), Multicenter Hemophilia Cohort Study (MHCS), San Francisco City Cohort (SFCC), ALIVE Study. Science 1997, 277(5328):959-965.

16. Dragic T, Litwin V, Allaway GP, Martin SR, Huang Y, Nagashima KA, Cayanan C, Maddon PJ, Koup RA, Moore JP, et al: HIV-1 entry into CD4 ${ }^{+}$cells is mediated by the chemokine receptor CC-CKR-5. Nature (London) 1996, 381:667-673.

17. Hladik F, Liu H, Speelmon E, Livingston-Rosanoff D, Wilson S, Sakchalathorn P, Hwangbo Y, Greene B, Zhu T, McElrath MJ: Combined effect of CCR5Delta32 heterozygosity and the CCR5 promoter polymorphism -2459 A/ $\mathrm{G}$ on CCR5 expression and resistance to human immunodeficiency virus type 1 transmission. J Virol 2005, 79(18):11677-11684.

18. Mummidi S, Ahuja SS, Gonzalez E, Anderson SA, Santiago EN, Stephan KT, Craig FE, O'Connell P, Tryon V, Clark RA, et al: Genealogy of the CCR5 locus and chemokine system gene variants associated with altered rates of HIV-1 disease progression. Nat Med 1998, 4(7):786-793.

19. O'Brien SJ, Nelson GW: Human genes that limit AIDS. Nature Genet 2004, 36(6):565-574.

20. Jeannet M, Sztajzel R, Carpentier N, Hirschel B, Tiercy JM: HLA antigens are risk factors for development of AIDS. J Acquir Immune Defic Syndr 1989, 2(1):28-32.

21. Nieto G, Barber $Y$, Rubio MC, Rubio M, Fibla J: Association between AIDS disease progression rates and the Fok-I polymorphism of the VDR gene in a cohort of HIV-1 seropositive patients. J Steroid Biochem Mol Biol 2004, 89-90(1-5):199-207.
22. Paxton WA, Kang S: Chemokine receptor allelic polymorphisms: relationships to HIV resistance and disease progression. Semin Immunol 1998, 10(3):187-194.

23. Ballana E, Senserrich J, Pauls E, Faner R, Mercader JM, Uyttebroeck F, Palou E, Mena MP, Grau E, Clotet B, et al: ZNRD1 (zinc ribbon domain-containing 1 ) is a host cellular factor that influences HIV-1 replication and disease progression. Clin Infect Dis 2010, 50(7):1022-1032.

24. Ciccosanti F, Corazzari M, Soldani F, Matarrese P, Pagliarini V, ladevaia V, Tinari A, Zaccarelli M, Perfettini $J$, Malorni W, et al: Proteomic analysis identifies prohibitin down-regulation as a crucial event in the mitochondrial damage observed in HIV-infected patients. Antivir Ther 2010, 15(3):377-390.

25. Melendez LM, Colon K, Rivera L, Rodriguez-Franco E, Toro-Nieves D: Proteomic analysis of HIV-infected macrophages. J Neuroimmune Pharmacol 2011, 6(1):89-106.

26. Ringrose $J H$, Jeeninga RE, Berkhout B, Speijer D: Proteomic studies reveal coordinated changes in T-cell expression patterns upon infection with human immunodeficiency virus type 1. J Virol 2008, 82(9):4320-4330.

27. Zhang X, Daucher M, Baeza J, Kim CW, Russell R, Kottilil S: Human immunodeficiency virus enhances hepatitis $C$ virus replication by differential regulation of IFN and TGF family genes. J Med Virol 2012, 84(9):1344-1352.

28. Geiss GK, Bumgarner RE, An MC, Agy MB, Wout AB V 't, Hammersmark E, Carter VS, Upchurch D, Mullins JI, Katze MG: Large-scale monitoring of host cell gene expression during HIV-1 infection using cDNA microarrays. Virology 2000, 266(1):8-16.

29. Ryo A, Suzuki Y, Arai M, Kondoh N, Wakatsuki T, Hada A, Shuda M, Tanaka K, Sato C, Yamamoto M, et al: Identification and characterization of differentially expressed mRNAs in HIV type 1-infected human T cells. AIDS Res Hum Retroviruses 2000, 16(10):995-1005.

30. Vahey MT, Nau ME, Jagodzinski LL, Yalley-Ogunro J, Taubman M, Michael $N L$, Lewis MG: Impact of viral infection on the gene expression profiles of proliferating normal human peripheral blood mononuclear cells infected with HIV type 1 RF. AIDS Res Hum Retroviruses 2002, 18(3):179-192.

31. Barnes MR, Deharo S, Grocock RJ, Brown JR, Sanseau P: The micro RNA target paradigm: a fundamental and polymorphic control layer of cellular expression. Expert Opin Biol Ther 2007, 7(9):1387-1399.

32. Carthew RW, Sontheimer EJ: Origins and Mechanisms of miRNAs and siRNAs. Cell 2009, 136(4):642-655

33. Chua JH, Armugam A, Jeyaseelan K: MicroRNAs: biogenesis, function and applications. Curr Opin Mol Ther 2009, 11(2):189-199.

34. Barik S: Silence of the transcripts: RNA interference in medicine. $J \mathrm{Mol}$ Med 2005, 83(10):764-773.

35. Amariglio N, Rechavi G: A-to-I RNA editing: a new regulatory mechanism of global gene expression. Blood Cells Mol Dis 2007, 39(2):151-155.

36. Gomase VS, Parundekar AN: microRNA: human disease and development. Int J Bioinform Res Appl 2009, 5(5):479-500.

37. Fritz JH, Girardin SE, Philpott DJ: Innate immune defense through RNA interference. Science's STKE: signal transduction knowledge environment 2006, 2006(339):pe27.

38. Carissimi C, Fulci V, Macino G: MicroRNAs: novel regulators of immunity. Autoimmun Rev 2009, 8(6):520-524.

39. O'Connell RM, Rao DS, Chaudhuri AA, Baltimore D: Physiological and pathological roles for microRNAs in the immune system. Nat Rev Immunol 2010, 10(2):111-122

40. Wang X, Ye L, Hou W, Zhou Y, Wang YJ, Metzger DS, Ho WZ: Cellular microRNA expression correlates with susceptibility of monocytes/ macrophages to HIV-1 infection. Blood 2009, 113(3):671-674.

41. Houzet L, Yeung ML, de Lame V, Desai D, Smith SM, Jeang KT: MicroRNA profile changes in human immunodeficiency virus type 1 (HIV-1) seropositive individuals. Retrovirology 2008, 5:118.

42. Van den Bergh R, Florence E, Vlieghe E, Boonefaes T, Grooten J, Houthuys E, Tran HT, Gali Y, De Baetselier P, Vanham G, et al: Transcriptome analysis of monocyte-HIV interactions. Retrovirology 2010, 7:53.

43. Wu JQ, Sasse TR, Wolkenstein G, Conceicao V, Saksena MM, Soedjono M, Perera SS, Wang B, Dwyer DE, Saksena NK: Transcriptome analysis of primary monocytes shows global down-regulation of genetic networks in HIV viremic patients versus long-term non-progressors. Virology 2013, 435(2):308-319.

44. Imbeault M, Giguere K, Ouellet M, Tremblay MJ: Exon level transcriptomic profiling of HIV-1-infected CD4(+) T cells reveals virus-induced genes 
and host environment favorable for viral replication. PLOS Pathog 2012, 8(8):e1002861.

45. Rotger M, Dang KK, Fellay J, Heinzen EL, Feng S, Descombes P, Shianna KV Ge D, Gunthard HF, Goldstein DB, et al: Genome-wide mRNA expression correlates of viral control in CD4+ T-cells from HIV-1-infected individuals. PLoS Pathog 2010, 6(2):e1000781.

46. Witwer KW, Watson AK, Blankson JN, Clements JE: Relationships of PBMC microRNA expression, plasma viral load, and CD4+ T-cell count in HIV-1 -infected elite suppressors and viremic patients. Retrovirology 2012, 9:5.

47. Chiang K, Sung TL, Rice AP: Regulation of cyclin T1 and HIV-1 Replication by microRNAs in resting CD4+ T lymphocytes. J Virol 2012, 86(6):3244-3252

48. Bignami F, Pilotti E, Bertoncelli L, Ronzi P, Gulli M, Marmiroli N, Magnani G, Pinti M, Lopalco L, Mussini C, et al: Stable changes in CD4+ T lymphocyte miRNA expression after exposure to HIV-1. Blood 2012, 119(26):6259-6267.

49. Zhou L, Pupo GM, Gupta P, Liu B, Tran SL, Rahme R, Wang B, Rua R, Rizos H, Carroll $A$, et al: A parallel genome-wide mRNA and microRNA profiling of the frontal cortex of HIV patients with and without HIV-associated dementia shows the role of axon guidance and downstream pathways in HIV-mediated neurodegeneration. BMC Genomics 2012, 13:677.

50. Chang ST, Thomas MJ, Sova P, Green RR, Palermo RE, Katze MG: NextGeneration Sequencing of Small RNAs from HIV-Infected Cells Identifies Phased microRNA Expression Patterns and Candidate Novel microRNAs Differentially Expressed upon Infection. mBio 2013, 4:1.

51. Jiang J, Lee E, Piper M, Marsh C, Schmittgen T: High-Throughput profiling of mature microRNA by real time PCR. RNA interference Techniques, Neuromethods 2011, 58:113-121.

52. Qi Y, Cui L, Ge Y, Shi Z, Zhao K, Guo X, Yang D, Yu H, Cui L, Shan Y, et al: Altered serum microRNAs as biomarkers for the early diagnosis of pulmonary tuberculosis infection. BMC Infect Dis 2012, 12:384.

53. Git A, Dvinge H, Salmon-Divon M, Osborne M, Kutter C, Hadfield J, Bertone $P$, Caldas C: Systematic comparison of microarray profiling, real-time PCR, and next-generation sequencing technologies for measuring differential microRNA expression. RNA 2010, 16(5):991-1006.

54. Wang B, Howel P, Bruheim S, Ju J, Owen LB, Fodstad O, Xi Y: Systematic evaluation of three microRNA profiling platforms: microarray, beads array, and quantitative real-time PCR array. PLoS One 2011, 6(2):e17167.

55. Backes C, Meese E, Lenhof HP, Keller A: A dictionary on microRNAs and their putative target pathways. Nucleic Acids Res 2010, 38(13):4476-4486.

56. Bartel DP: MicroRNAs: target recognition and regulatory functions. Cell 2009, 136(2):215-233.

57. Shalgi $R$, Lieber $D$, Oren M, Pilpel $Y$ : Global and local architecture of the mammalian microRNA-transcription factor regulatory network. PLoS Comput Biol 2007, 3(7):e131.

58. Wienholds E, Plasterk RH: MicroRNA function in animal development FEBS Lett 2005, 579(26):5911-5922.

59. Le HS, Bar-Joseph Z: Inferring Interaction Networks using the IBP applied to microRNA Target Prediction. Advances in Neural Information Processing Systems 2011, 2011:235-243.

60. Telenti A, Johnson WE: Host Genes Important to HIV Replication and Evolution. Cold Spring Harb Perspect Med 2012, 2(4):a007203.

61. Mehla R, Ayyavoo V: Gene array studies in HIV-1 infection. Curr HIV/AIDS Rep 2012, 9(1):34-43.

62. Kim YK, Kim VN: Processing of intronic microRNAs. EMBO J 2007, 26(3):775-783

63. Ballarino M, Pagano F, Girardi E, Morlando M, Cacchiarelli D, Marchioni M Proudfoot NJ, Bozzoni l: Coupled RNA processing and transcription of intergenic primary microRNAs. Mol Cell Biol 2009, 29(20):5632-5638.

64. Morlando M, Ballarino M, Gromak N, Pagano F, Bozzoni I, Proudfoot NJ: Primary microRNA transcripts are processed co-transcriptionally. Nat Struct Mol Biol 2008, 15(9):902-909.

65. Allantaz F, Cheng DT, Bergauer T, Ravindran P, Rossier MF, Ebeling M, Badi L, Reis $B$, Bitter $H$, D'Asaro M, et al: Expression profiling of human immune cell subsets identifies miRNA-mRNA regulatory relationships correlated with cell type specific expression. PLoS One 2012, 7(1):e29979.

66. Wu H, Neilson JR, Kumar P, Manocha M, Shankar P, Sharp PA, Manjunath N: miRNA profiling of naive, effector and memory CD8 T cells. PLoS One 2007, 2(10):e1020

67. Huang J, Wang F, Argyris E, Chen K, Liang Z, Tian H, Huang W, Squires K, Verlinghieri $\mathrm{G}$, Zhang $\mathrm{H}$ : Cellular microRNAs contribute to HIV-1 latency in resting primary CD4+ T lymphocytes. Nat Med 2007, 13(10):1241-1247.
68. Chiang $K$, Liu H, Rice AP: miR-132 enhances HIV-1 replication. Virology 2013, 438(1):1-4.

69. Swaminathan S, Suzuki K, Seddiki N, Kaplan W, Cowley MJ, Hood CL, Clancy $J$, Murray DD, Mendez C, Gelgor L, et al: Differential regulation of the Let7 family of microRNAs in CD4+ T cells alters IL-10 expression. J Immunol 2012, 188(12):6238-6246.

70. Sung TL, Rice AP: miR-198 inhibits HIV-1 gene expression and replication in monocytes and its mechanism of action appears to involve repression of cyclin T1. PLoS Pathog 2009, 5(1):e1000263.

71. Sisk JM, Clements JE, Witwer KW: miRNA profiles of monocyte-lineage cells are consistent with complicated roles in HIV-1 restriction. Viruses 2012, 4(10):1844-1864.

72. Santhakumar D, Forster T, Laqtom NN, Fragkoudis R, Dickinson P, AbreuGoodger C, Manakov SA, Choudhury NR, Griffiths SJ, Vermeulen A, et al: Combined agonist-antagonist genome-wide functional screening identifies broadly active antiviral microRNAs. Proc Natl Acad Sci U S A 2010, 107(31):13830-13835.

73. Sonkoly E, Stahle M, Pivarcsi A: MicroRNAs and immunity: novel players in the regulation of normal immune function and inflammation. Semin Cancer Biol 2008, 18(2):131-140.

74. Li L, Chen XP, Li YJ: MicroRNA-146a and human disease. Scand J Immunol 2010, 71(4):227-231

75. Lukiw WJ, Dua P, Pogue Al, Eicken C, Hill JM: Upregulation of micro RNA146a (miRNA-146a), a marker for inflammatory neurodegeneration, in sporadic Creutzfeldt-Jakob disease (sCJD) and Gerstmann-StrausslerScheinker (GSS) syndrome. J Toxicol Environ Health A 2011, 74(22-24):1460-1468

76. Zhang Y, Wei W, Cheng N, Wang K, Li B, Jiang X, Sun S: Hepatitis C Virusinduced upregulation of miR-155 promotes hepatocarcinogenesis by activating Wnt signaling. Hepatology 2012, 56(5):1631-1640.

77. Xie Q, Chen X, Lu F, Zhang T, Hao M, Wang Y, Zhao J, McCrae MA, Zhuang $\mathrm{H}$ : Aberrant expression of microRNA 155 may accelerate cell proliferation by targeting sex-determining region $\mathrm{Y}$ box 6 in hepatocellular carcinoma. Cancer 2012, 118(9):2431-2442.

78. Wie SH, Du P, Luong TQ, Rought SE, Beliakova-Bethell N, Lozach J, Corbeil J, Kornbluth RS, Richman DD, Woelk CH: HIV Downregulates InterferonStimulated Genes in Primary Macrophages. J Interferon Cytokine Res 2013, 33(2):90-95

79. Matsumoto T, Miike T, Nelson RP, Trudeau WL, Lockey RF, Yodoi J: Elevated serum levels of IL-8 in patients with HIV infection. Clin Exp Immunol 1993, 93(2):149-151.

80. Stone SF, Price P, Keane NM, Murray RJ, French MA: Levels of IL-6 and soluble IL- 6 receptor are increased in HIV patients with a history of immune restoration disease after HAART. HIV Med 2002, 3(1):21-27.

81. Venkatachari NJ, Buchanan WG, Ayyavoo V: Human immunodeficiency virus (HIV-1) infection selectively downregulates PD-1 expression in infected cells and protects the cells from early apoptosis in vitro and in vivo. Virology 2008, 376(1):140-153.

82. Schafer EA, Venkatachari NJ, Ayyavoo V: Antiviral effects of mifepristone on human immunodeficiency virus type-1 (HIV-1): targeting Vpr and its cellular partner, the glucocorticoid receptor (GR). Antiviral Res 2006, 72(3):224-232

83. Janket ML, Manickam P, Majumder B, Thotala D, Wagner M, Schafer EA Collman RG, Srinivasan A, Ayyavoo V: Differential regulation of host cellular genes by HIV-1 viral protein R (Vpr): cDNA microarray analysis using isogenic virus. Biochem Biophys Res Commun 2004, 314(4):1126-1132.

doi:10.1186/1471-2334-13-250

Cite this article as: Duskova et al: MicroRNA regulation and its effects on cellular transcriptome in Human Immunodeficiency Virus-1 (HIV-1) infected individuals with distinct viral load and CD4 cel counts. BMC Infectious Diseases 2013 13:250. 